\title{
Equilibrium Theory with Asymmetric Information and with Infinitely many Commodities
}

\author{
Konrad Podczeck* Nicholas C. Yannelis ${ }^{\dagger}$
}

December 23, 2005

\begin{abstract}
The traditional deterministic general equilibrium theory with infinitely many commodities cannot cover economies with private information constraints on the consumption sets. We bring the level of asymmetric information equilibrium theory at least at par with that of the deterministic. In particular, we establish results on equilibrium existence for exchange economies with asymmetric (differential) information and with an infinite dimensional commodity space. Two settings are treated. In the first, the commodity space is an ordered normed space whose positive cone has a non-empty interior, while in the second it is a locally convex-solid Riesz space whose positive cone may have empty interior. Preferences are not assumed to be monotone, complete, or transitive. Our new equilibrium existence theorems for asymmetric information economies not only include, as a special case, classical results, e.g. Bewley (1972), Mas-Colell (1986), among others, but also complement and extend the techniques and some results in Aliprantis, Tourky, and Yannelis (2001). Furthermore, they imply new existence results for the core of an economy with asymmetric information.
\end{abstract}

\section{Introduction}

Uncertainty was introduced in equilibrium theory by Arrow and Debreu. Both authors realized (see for example Chapter 7 of the classical treatise of Debreu, "Theory of Value") that if the exogenous uncertainty is described by a set which denotes the states of nature of the world and agents' characteristics, i.e. preferences and initial endowments, become random (state dependent), then the classical equilibrium results on existence and optimality of the Walrasian equilibrium continue to hold.

\footnotetext{
*Institut für Wirtschaftswissenschaften, Universität Wien, Hohenstaufengasse 9, A-1010 Wien, Austria. E-mail: konrad.podczeck@univie.ac.at

${ }^{\dagger}$ Department of Economics, University of Illinois at Urbana-Champaign, IL 61820, USA. Email: nyanneli@uiuc.edu
} 
This is the so called "state contingent" model which captures the meaning of contacts (or trades) under uncertainty. However, this model doesn't allow for trades to be made under asymmetric information as agents uncertainty is common. In a seminal paper, Radner (1968) allowed, in addition to the random preferences and initial endowments, each agent to have her own private information set which was described by a partition of an exogenously given set of states of nature. In this model optimal choices reflect the private informations of the agents as net trades of an agent are measurable with respect to her private information partition, i.e. measurable with respect to the $\sigma$-algebra that her information partition generates. Furthermore, the market clearing occurs in the sense that total consumption is equal (or less than equal, which amounts to free disposal) to the total initial endowment for each state of nature. Thus, Radner introduced asymmetric information in the Arrow-Debreu model.

Concerning the assumption of free disposal, Radner himself realized that this assumption may be problematic in the context of asymmetric information. ${ }^{1}$ Indeed, as it was shown in Glycopantis, Muir, and Yannelis (2003), the free disposal assumption may destroy the incentive compatibility of the Walrasian equilibrium and thus the resulting trades (contracts) need not be incentive compatible. Also, the free disposal assumption results in allocations which are not consistent with Bayesian rationality, i.e., they may not be implementable as a perfect Bayesian equilibrium of an extensive form game. On the other hand, it is easy to construct examples of well behaved differential information economies where no Walrasian equilibrium exists with positive prices and without free disposal (see Glycopantis et al., 2003, or Section 4 below).

The main purpose of this paper is to extend the theory of differential information economies to infinite dimensional commodities spaces. In particular, we prove the existence of a Walrasian equilibrium for an economy with asymmetric (differential) information. The commodity spaces treated are general enough to include most infinite dimensional spaces appearing in equilibrium theory. Moreover, we allowed for very general preferences, i.e., preferences need not be transitive or complete. In fact, we could even allow for interdependent preferences. Furthermore, our results not only include the expected utility framework and the corresponding Walrasian expectations equilibrium notion as a special case, but also dispense with the assumption of free disposal. It turns out that, by dispensing with the free disposal assumption, we are able to guarantee the incentive compatibility of a Walrasian expectations equilibrium.

Despite the fact that infinite dimensional commodity spaces have been introduced in order to capture the meaning of uncertainty or commodity differentiation or infinite time horizon, none of the existing infinite dimensional com-

\footnotetext{
${ }^{1}$ To quote Radner on this point: “... the assumption of free disposal is inappropriate here, at least in its usual form; even though a given act $\alpha$ is compatible with an information structure $S$, the set of acts $\leq \alpha$ (in the vector sense) will typically contain acts that are not compatible with $S . "$
} 
modity spaces models allow for asymmetric information and no free disposal simultaneously. Our results indicate that such a generalization is possible and therefore we bring the level of asymmetric information equilibrium theory at par with that of the deterministic equilibrium one.

In view of the counterexample in Glycopantis et al. (2003), i.e., no Walrasian expectations equilibrium exists without free disposal and positive prices, in order to dispense with free disposal we allow for non-positive prices. However, there are several technical difficulties which need to be bypassed. First, even in finite dimensional commodity spaces, one cannot adopt the standard assumption that endowments are in the interior of the consumption sets since consumption sets are very "thin" in the presence of asymmetric information and may have no interior points. Thus, to allow for non ordered preferences and no free disposal, neither the Shafer (1976) nor the Won and Yannelis (2005) results apply directly and we need to start from "scratch."

Furthermore, the infinite dimensional standard arguments and results are not directly applicable. The reason is that the (informationally constrained) consumption sets do not coincide with the positive cone of the commodity space, and also are not upper comprehensive. In particular, even when the positive cone of the commodity space has non-empty interior, this is not so for the consumption sets. Moreover, for the case where the positive cone of the commodity space has an empty interior, properness assumptions on preferences and techniques like the Riesz-Kantorovich formula (see e.g. Aliprantis, Tourky, and Yannelis, 2000) do not work immediately when trying to use the standard approach of obtaining an equilibrium by a pass from an equilibrium relative to the restriction of the economy to the order ideal generated by the aggregate endowment. In particular, non-positivity of price systems is a problem here, so that some intermediate constructions and new arguments are necessary. Thus, our new equilibrium existence results not only include, as a special case, generalized versions of the classical deterministic results, e.g. Bewley (1972) and Mas-Colell (1986), but also complement and extend the techniques and some results in Aliprantis, Tourky, and Yannelis (2001).

The paper is organized as follows. In the next section, some notational and terminological conventions are settled. In Section 3 the model and the results are presented. Two settings will be treated. In the first one, the commodity space is an ordered normed space whose positive cone has a non-empty interior, while in the second one it is a locally convex-solid Riesz space whose positive cone may have empty interior. Actually, the equilibrium existence result of the first setting will serve as an essential tool to establish the existence result of the second setting. In Section 4 we argue that the expected utility model of differential information economies is a special case of our general framework. Finally, Section 5 contains the proofs. 


\section{Notation and terminology}

As usually, the term ordered vector space means a real vector space $E$ endowed with a partial ordering $\geq$ (i.e. $\geq$ is a reflexive, transitive, and anti-symmetric relation) such that $x \geq y$ entails $x+z \geq y+z$, and $x \geq 0$ entails $\lambda x \geq 0$, for $x$, $y, z \in E$ and $\lambda \in \mathbb{R}$ with $\lambda>0$. We will write $x \leq y$ to mean $y \geq x$.

Let $E$ be an ordered vector space. We write $E_{+}$for the positive cone of $E$, i.e. $E_{+}=\{x \in E: x \geq 0\}$. Thus $E_{+}$is a convex cone satisfying $E_{+} \cap-E_{+}=\{0\}$. Further, for $x, y \in E$ with $x \leq y$, we denote by $[x, y]$ the order interval $\{z \in E: x \leq z \leq y\}$.

By an ordered normed space we mean an ordered vector space $E$ endowed with a norm such that the positive cone $E_{+}$is closed.

Recall that a Riesz space (or vector lattice) is an ordered vector space $E$ such that for any $x, y \in E$ the set $\{x, y\}$ has a supremum and an infimum in $E$ (for the ordering $\geq$ of $E$ ). Let $E$ be a Riesz space. Given $x, y \in E$, the expressions $x^{+}$, $x^{-},|x|, x \vee y, x \wedge y$, and $x \perp y$ have the usual meaning, and we will write $L(x)$ for the order ideal in $E$ generated by $x$. Thus, if $x \in E_{+}$then

$$
L(x)=\bigcup_{n=1}^{\infty}[-n x, n x]=\{z \in E:|z| \leq n x \text { for some } n \in \mathbb{N}\} .
$$

An element $x \geq 0$ of a Riesz space $E$ is said to be an order unit if $L(x)=E$, and if $E$ is a locally convex-solid Riesz space, then an element $x \in E_{+}$is said to be a quasi-interior point of $E_{+}$if $L(x)$ is dense in $E$. (Of course, an order unit in a locally convex-solid Riesz space $E$ is a quasi-interior point of $E_{+}$. The converse does not hold in general.)

When a product $E^{\Omega}$ is involved, where $\Omega$ is a non-empty finite set and $E$ is both an ordered vector space and a topological vector space, then $E^{\Omega}$ is always regarded as endowed with the product topology and the product ordering. With this convention, if $E$ is a Hausdorff locally convex-solid Riesz space, then so is $E^{\Omega}$, and if $E$ is an ordered normed space, then the topology of $E^{\Omega}$ is normable so that $E^{\Omega}$ becomes an ordered normed space.

Given a topological vector space $E$, we denote by $E^{*}$ the topological dual space of $E$, i.e. the space of all continuous linear functions from $E$ into $\mathbb{R}$. For a product $E^{\Omega}$, the topological dual is denoted by $E^{\Omega, *}$.

Finally, for a subset $A$ of a topological space, int $A$ denotes the interior of $A$, and $\mathrm{c} \ell A$ the closure of $A$.

\section{The model and the results}

Let $E$ be a Hausdorff locally convex-solid Riesz space, or an ordered normed vector space (whose ordering need not be a lattice ordering). Let $\Omega$ be a nonempty finite set of states of nature. Given a partition $\mathcal{P}$ of $\Omega$, we will say that an element $x \in E^{\Omega}$ is $\mathcal{P}$-measurable if $S \in \mathcal{P}$ and $s, s^{\prime} \in S$ imply $x(s)=x\left(s^{\prime}\right)$. 
A differential information economy $\mathcal{E}$ with finitely many agents and commodity space $E^{\Omega}$ is a family $\mathcal{E}=\left[\left(\mathcal{H}_{i}, P_{i}, w_{i}\right)_{i \in I}\right]$ where

$-I=\{1, \ldots, n\}$ is the finite set of agents;

- for each $i \in I, \mathcal{H}_{i}$ is the private information partition of $\Omega$;

- for each $i \in I$, the consumption set is the set

$$
X_{i}=\left\{x \in E_{+}^{\Omega}: x \text { is } \mathcal{H}_{i} \text {-measurable }\right\}
$$

- for each $i \in I, P_{i}: X_{i} \rightarrow 2^{X_{i}}$ is the (strict) preference relation;

- for each $i \in I, w_{i} \in X_{i}$ is the initial endowment;

and such that

$-\sum_{i \in I} w_{i} \neq 0$.

(Preferences coming from expected utility are a special case of this setting; see Section 4 below.) Note that this definition implies that each consumption set $X_{i}$ is a closed convex cone in $E_{+}^{\Omega}$.

An allocation for the economy $\mathcal{E}$ is a list $\left(x_{i}\right)_{i \in I}$ where $x_{i} \in X_{i}$ for each $i \in I$. The allocation $\left(x_{i}\right)_{i \in I}$ is said to be feasible if $\sum_{i \in I} x_{i}=\sum_{i \in I} w_{i}$; it is said to be individually rational if $w_{i} \notin P_{i}\left(x_{i}\right)$ for each $i \in I$; it is said to be Pareto optimal if it is feasible and if there is no feasible allocation $\left(x_{i}^{\prime}\right)_{i \in I}$ with $x_{i}^{\prime} \in P_{i}\left(x_{i}\right)$ for each $i \in I$. A quasi-equilibrium for $\mathcal{E}$ is a pair $\left(\left(x_{i}\right)_{i \in I}, p\right)$ where $\left(x_{i}\right)_{i \in I}$ is a feasible allocation and $p \in E^{\Omega, *}$ is a price system with $p \neq 0$ such that for each $i \in I$, $p x_{i} \leq p w_{i}$ and whenever $y \in P_{i}\left(x_{i}\right)$ then $p y \geq p w_{i}$. The quasi-equilibrium $\left(\left(x_{i}\right)_{i \in I}, p\right)$ will be called individually rational if the allocation $\left(x_{i}\right)_{i \in I}$ is individually rational, and it will be called non-trivial if some agents have income, i.e. if $p w_{i}>\inf \left\{p y: y \in X_{i}\right\}$ holds for some $i \in I$. Finally, an equilibrium is a quasi-equilibrium $\left(\left(x_{i}\right)_{i \in I}, p\right)$ where $y \in P_{i}\left(x_{i}\right)$ actually implies $p y>p w_{i}$.

Note that there is no free disposal assumption embodied in the notion of feasibility and that a (quasi-) equilibrium price system is required to be continuous for the topology of $E^{\Omega}$. Also note that the term "Pareto optimal" must be interpreted relative to the given information structure of the economy. Finally note that since consumption sets are convex, if the economy satisfies a suitable irreducibility condition and preferences have open upper sections-which is implied by Assumption (A5) below-then every non-trivial quasi-equilibrium will in fact be an equilibrium.

We will consider the following assumptions.

(A1) For every $i \in I$ and each $x \in X_{i}, x \notin P_{i}(x)$ (irreflexivity).

(A2) For every $i \in I$ and each $x \in X_{i}$, the set $P_{i}(x)$ is convex.

(A3) Whenever $\left(x_{i}\right)_{i \in I}$ is a feasible and individually rational allocation, then $P_{i}\left(x_{i}\right) \neq \varnothing$ for each $i \in I$ (non-satiation of preferences at feasible and individually rational allocations). 
(A4) Whenever $\left(x_{i}\right)_{i \in I}$ is a Pareto optimal and individually rational allocation, then $x_{i} \in \mathrm{c} \ell P_{i}\left(x_{i}\right)$ for each $i \in I$ (local non-satiation of preferences at Pareto optimal and individually rational allocations).

For the next assumption we introduce the following notation: If $\eta$ is some topology on (the set underlying) $E$, which may be different from the original topology of $E$, then $\eta^{\Omega}$ denotes the product topology $\eta \times \eta \times \cdots \times \eta$ on $E^{\Omega}$.

(A5) There is a Hausdorff linear topology $\eta$ on $E$ such that all order intervals in $E$ are $\eta$-compact and such that for each $i \in I, P_{i}$ is $\eta^{\Omega_{-} \text {"original topology }}$ of $E^{\Omega}$ "-continuous, i.e. $P_{i}$ has a relatively open graph in $X_{i} \times X_{i}$ for the product topology $\eta^{\Omega} \times$ "original topology of $E^{\Omega}$ ".

See below for examples of spaces for which the hypothesis on order intervals stated in this assumption is satisfied. We refer to Yannelis and Zame (1986) for a discussion of formulating a continuity assumption on preferences in terms of a "mixed topology."

Our first existence result addresses the case where the space $E$ is actually an ordered normed space whose positive cone $E_{+}$has a non-empty interior. In this case, the above assumptions can be shown to be sufficient to guarantee the existence of a quasi-equilibrium. Of course, they are not sufficient to guarantee the existence of a quasi-equilibrium that is non-trivial. To get a non-trivial quasiequilibrium, we will use the following assumption in addition.

(A6) There is a feasible allocation $\left(x_{i}\right)_{i \in I}$ such that for each $i \in I$ and each $s \in \Omega, x_{i}(s) \in \operatorname{int} E_{+}$.

The interpretation of this assumption is that there be a feasible (in particular, informationally feasible) allocation in which, in each single state, each agent gets a commodity bundle containing every commodity. Note that in the finite dimensional case $E=\mathbb{R}^{\ell}$, (A6) says exactly that there be a feasible allocation in which in each state, each agent gets a quantity $>0$ of every commodity. A similar assumption was used in Radner (1968). Note also that (A6) automatically holds would we assume that for each $i \in I$ and each $s \in \Omega$, the endowment $w_{i}(s)$ belongs to int $E_{+}$.

Theorem 1. Let $E$ be an ordered normed space such that $E_{+}$has a non-empty interior, let $\Omega$ be a non-empty finite set of states, and let $\mathcal{E}=\left[\left(\mathcal{H}_{i}, P_{i}, w_{i}\right)_{i \in I}\right]$ be a differential information economy with commodity space $E^{\Omega}$. Suppose that (A1) to (A6) hold for $\mathcal{E}$. Then $\mathcal{E}$ has a non-trivial individually rational quasi-equilibrium.

(See Section 5.2 for the proof.) Theorem 1 extends results of Bewley (1972) and Florenzano (1983) to the asymmetric information context.

Next we will address the case where $E$ is a locally convex-solid Riesz space whose positive cone $E_{+}$may have an empty interior. It is a well known fact that without some properness hypotheses on preferences, equilibrium existence may 
fail even in standard complete information economies when the positive cone of the commodity space has empty interior. In this paper, we will use the following properness notion, which was introduced in Aliprantis, Tourky, and Yannelis (2000) under the name " $v$-proper," and which we will call "ATY-proper" here, the "ATY" standing for the mentioned authors.

Definition 1. The preference relation $P_{i}$ is said to be ATY-proper at $x_{i} \in X_{i}$ if there is a convex subset $\widetilde{P}_{i}\left(x_{i}\right)$ of $E^{\Omega}$, with non-empty interior, such that both $\widetilde{P}_{i}\left(x_{i}\right) \cap X_{i}=P_{i}\left(x_{i}\right)$ and $\left(\operatorname{int} \widetilde{P}_{i}\left(x_{i}\right)\right) \cap X_{i} \neq \varnothing$.

See also Remark 2 below. (The original name " $v$-proper" in Aliprantis et al. (2000) was in order to indicate that for a certain element $v$ of the commodity space, $x_{i}+v$ should belong to the intersection (int $\left.\widetilde{P}_{i}\left(x_{i}\right)\right) \cap X_{i}$. In this paper, we have no need to do so, and have therefore chosen a different name to avoid confusion.) We will assume:

(A7) Whenever $\left(x_{i}\right)_{i \in I}$ is a Pareto optimal and individually rational allocation, then $P_{i}$ is ATY-proper at $x_{i}$ for each $i \in I$.

For the following, recall from Section 2 that for an $x \in E_{+}, L(x)$ denotes the order ideal in $E$ generated by $x$. The next assumption is an adaptation of (A6) to the present setting where the positive cone of $E$ may have empty interior.

(A8) There is a feasible allocation $\left(x_{i}\right)_{i \in I}$ such that for each $i \in I$ and each $s \in \Omega, \sum_{j \in I} w_{j}(s) \in L\left(x_{i}(s)\right)$.

The interpretation of (A8) is that there be a feasible allocation such that in each single state, each agent gets a commodity bundle containing every commodity available in the aggregate. In the case where the interior of $E_{+}$is non-empty, in particular if $E=\mathbb{R}^{\ell}$, this assumption is implied by (A6), and it implies (A6) if in each state the aggregate endowment belongs to the interior of $E_{+}$.

We will also assume that the aggregate endowments in the single states do not vary too much across states.

(A9) There is an $\bar{e} \in E_{+}$such that $L\left(\sum_{i \in I} w_{i}(s)\right)=L(\bar{e})$ for each $s \in \Omega$,.

For spaces $E$ which contain order units, e.g. $E=\mathbb{R}^{\ell}$ or $E=L_{\infty}(\mu)$, one can assume that the aggregate endowment in each state is an order unit, which implies (A9). In particular, if the interior of $E_{+}$is non-empty and it is assumed that the aggregate endowment in each state is in the interior of $E_{+}$then (A9) holds. We remark that at the cost of some additional burden of notation in the proofs, (A9) can be dropped from the statements of Theorems 2 and 3 below.

We will first consider the case of spaces whose positive cones have quasiinterior points. ${ }^{2}$ A natural assumption is then:

\footnotetext{
${ }^{2}$ Recall from Section 2 that, for a locally convex-solid Riesz space $E$, an $x \in E_{+}$is called a quasi-interior point of $E_{+}$if the order ideal generated by $x$ is dense in $E$.
} 
(A10) $\sum_{i \in I} w_{i}(s)$ is a quasi-interior point of $E_{+}$for each $s \in \Omega$.

The class of locally convex-solid Riesz spaces whose positive cones contain quasi-interior points includes, in particular, those locally convex-solid Riesz spaces which have order units. Thus it includes $L_{\infty}(\mu)$ when endowed with the sup-norm topology as well as when endowed with the Mackey topology $\tau\left(L_{\infty}(\mu), L_{1}(\mu)\right)$. In particular, it includes $\ell_{\infty}$ with the sup-norm topology as well as with the Mackey topology $\tau\left(\ell_{\infty}, \ell_{1}\right)$. It also includes several classical Banach lattices that have no order units, e.g. the sequence spaces $c_{0}$ and $\ell_{p}, 1 \leq p<\infty$, as well as the Lebesgue spaces $L_{p}(\mu), 1 \leq p<\infty$, when $\mu$ is $\sigma$-finite.

Note that for all these spaces there exists some linear topology $\eta$ such that all order intervals are $\eta$-compact, as required in assumption (A5). In fact, for the spaces $c_{0}, \ell_{p}$, and $L_{p}(\mu), 1 \leq p<\infty$, the weak topology does the job, while for $L_{\infty}(\mu)$ and $\ell_{\infty}$ one can take the weak* topology with respect to $L_{1}(\mu)$ and $\ell_{1}$, respectively.

Theorem 2. Let E be a locally convex-solid Riesz space whose positive cone contains quasi-interior points, let $\Omega$ be a non-empty finite set of states, and let $\mathcal{E}=$ $\left[\left(\mathcal{H}_{i}, P_{i}, w_{i}\right)_{i \in I}\right]$ be a differential information economy with commodity space $E^{\Omega}$. Suppose that (A1) to (A5) and (A7) to (A10) hold for $\mathcal{E}$. Then $\mathcal{E}$ has a non-trivial quasi-equilibrium.

(See Section 5.3 for the proof.) Theorem 2 extends results of Yannelis and Zame (1986) and Araujo and Monteiro (1989) to asymmetric information economies (modulo that the properness assumption there is slightly different; cf. Remark 2).

Finally, we address the case where Assumption (A10) need not hold, and in particular the case where the positive cone of the commodity space may not contain quasi-interior points at all. An example for this latter case is the space $c a(K)$ of bounded regular Borel measures on a compact Hausdorff space $K$, endowed with the total variation norm. Recall that order intervals in $c a(K)$ are weakly compact, so that Assumption (A5) is satisfiable.

Note that under (A10) and (A9), for each $i \in I$ the intersection of $X_{i}$ with $L\left(\sum_{i \in I} w_{i}\right)$, the order ideal in $E^{\Omega}$ generated by the total endowment $\sum_{i \in I} w_{i}$, is dense in $X_{i}$, so that the sets $\widetilde{P}_{i}\left(x_{i}\right)$ from the definition of ATY-properness must actually satisfy $\left(\operatorname{int} \widetilde{P}_{i}\left(x_{i}\right)\right) \cap X_{i} \cap L\left(\sum_{i \in I} w_{i}\right) \neq \varnothing$. For the general case where (A10) may not hold, we have to assume this property explicitly:

(A7') Whenever $\left(x_{i}\right)_{i \in I}$ is a Pareto optimal and individually rational allocation, then for each $i \in I, P_{i}$ is ATY-proper at $x_{i}$ such that the set $\widetilde{P}_{i}\left(x_{i}\right)$ from the definition of ATY-properness satisfies $\left(\operatorname{int} \widetilde{P}_{i}\left(x_{i}\right)\right) \cap X_{i} \cap L\left(\sum_{i \in I} w_{i}\right) \neq \varnothing$.

Similarly, we have to adjust the statements of (A3) and (A4) in the following way.

(A3') Whenever $\left(x_{i}\right)_{i \in I}$ is a feasible and individually rational allocation, then $P_{i}\left(x_{i}\right) \cap L\left(\sum_{i \in I} w_{i}\right) \neq \varnothing$ for each $i \in I$. 
(A4') Whenever $\left(x_{i}\right)_{i \in I}$ is a Pareto optimal and individually rational allocation, then $x_{i} \in \mathrm{c} \ell\left(P_{i}\left(x_{i}\right) \cap L\left(\sum_{i \in I} w_{i}\right)\right)$ for each $i \in I$.

We can now state the following theorem.

Theorem 3. Let $E$ be a locally convex-solid Riesz space, let $\Omega$ be a non-empty finite set of states, and let $\mathcal{E}=\left[\left(\mathcal{H}_{i}, P_{i}, w_{i}\right)_{i \in I}\right]$ be a differential information economy with commodity space $E^{\Omega}$. Suppose that (A1), (A2), (A3'), (A4'), (A5), (A7'), (A8) and (A9) hold for $\mathcal{E}$. Then $\mathcal{E}$ has a non-trivial quasi-equilibrium.

(See Section 5.4 for the proof.) For the special case of only one state of nature, Theorem 3 reduces to a generalized version of the classical result of Mas-Colell (1986), thus extending this latter result to the asymmetric information context.

We close this section with some remarks.

Remark 1. Open problems. In the special case of symmetric information (and in particular in the case of only one state of nature), the conclusion of Theorem 2 continues to hold when the requirement of ATY-properness in the statement of assumption (A7) is replaced by the weaker properness notion that results when the equality $\widetilde{P}_{i}\left(x_{i}\right) \cap X_{i}=P_{i}\left(x_{i}\right)$ in the definition of ATY-properness is replaced by the inclusion $\widetilde{P}_{i}\left(x_{i}\right) \cap X_{i} \subset P_{i}\left(x_{i}\right)$. (See e.g. Theorem 1 in Podczeck, 1996. ${ }^{3}$ ) We leave it as an open question whether, in the context of Theorem 2, this weaker properness notion would also be sufficient for the case of a differential information economy.

Further, we leave it as an open question whether Theorems 2 and 3 can be generalized to cover the case where the commodity space is just a vector lattice with a locally convex topology such that the topological dual space is a sublattice of the order dual of the commodity space, rather that an ideal in the order dual as implied by the hypothesis that the commodity space be locally convex-solid. This latter generalization would cover e.g. the case where, in each state, the commodity space is $c a(K)$ with a topology such that the price space becomes $C(K)$, the space of continuous functions on $K$.

Remark 2. The notion of $v$-properness (or ATY-properness as we have called it here) goes back to the notion of M-properness introduced by Tourky (1998). (See also Tourky, 1999.) A related properness notion can be found in Podczeck (1996) under the name "E-proper." Similarly to Mas-Colell's (1986) original notion of properness, E-properness is formulated in terms of open cones. It may be seen that, for a convex consumption set, if a preference relation is both $v$ proper and locally non-satiated at some point, then it is E-proper at this point. Theorem 2 continues to hold if ATY-properness in the statement of assumption (A7) is replaced by E-properness. It should also be noted that for a convex

\footnotetext{
${ }^{3}$ The F-properness assumption in Theorem 1 in Podczeck (1996) may be seen to be implied by the just indicated weakening of ATY-properness if Assumption (A4) holds and consumption sets are convex.
} 
valued and transitive preference relation $P_{i}: X_{i} \rightarrow 2^{X_{i}}$, and a consumption set $X_{i}$ being a convex cone, $v$-properness holds at every point $x \in X_{i}$ if $P_{i}$ is uniformly proper in the sense that there is an open convex cone $\Gamma_{i}$ in the commodity space with $\Gamma_{i} \cap X_{i} \neq \varnothing$ such that whenever $x \in X_{i}, \gamma \in \Gamma_{i}$, and $x+\gamma \in X_{i}$ then $x+\gamma \in P_{i}(x)$; cf. the argument in the third paragraph of Section 4.

Remark 3. Theorems 1 and 2 generalize to cover interdependent preferences, i.e. the situation where the preferences of an agent over her consumption set may depend on the consumption of other agents, so that preferences are given by correspondences $P_{i}: \prod_{j \in I} X_{j} \rightarrow 2^{X_{i}}$. Some changes in the statements of some of the assumptions, but only trivial changes of the proofs, mainly adjustments of notation, would become necessary. However, with interdependent preferences, the term "Pareto optimal" which is involved in assumptions (A4) and (A7) should be interpreted as conditional on the preferences corresponding to a given allocation.

\section{Expected utility models, incentive compatibility, and the core}

We will argue in this section that the expected utility model of differential information economies is captured by the setting presented in this paper. Indeed, with $E$ and $\Omega$ as in the previous section, suppose that for each agent $i \in I$ the consumption set in every state $s \in \Omega$ is equal to $E_{+}$and that his preferences over sure consumption bundles, i.e. points of $E_{+}$, are given by a state dependent utility function $u_{i}: \Omega \times E_{+} \rightarrow \mathbb{R}$. Moreover, suppose that each $i \in I$ is characterized by a probability function $\pi_{i} \in \mathbb{R}_{+}^{\Omega}$, with $\pi_{i}(s)>0$ for each $s \in \Omega$, where $\pi_{i}(s)$ represents agent $i$ 's subjective prior probability of the occurrence of state $s$. Assume that each agent forms (ex ante) preferences over random consumption vectors, i.e. elements of $E_{+}^{\Omega}$, according to expected utility. A differential information economy with commodity space $E^{\Omega}$ can then be described by a list $\mathcal{E}=\left[\left(\mathcal{H}_{i}, u_{i}, \pi_{i}, w_{i}\right)_{i \in I}\right]$, where $\mathcal{H}_{i}$ and $w_{i}$ have the same meaning as in the previous section. In the following, let such an economy $\mathcal{E}$ be given.

For each agent $i \in I$ and each random consumption bundle $x \in E^{\Omega}$, let $v_{i}(x)$ denote the expected utility of $x$ for $i$, i.e. $v_{i}(x)=\sum_{s \in \Omega} \pi_{i}(s) u_{i}(s, x(s))$. Thus, for each $i \in I$, the strict preference relation $P_{i}: X_{i} \rightarrow 2^{X_{i}}$ over the informationally feasible consumption set $X_{i}$ (defined as above via the private information partition $\mathcal{H}_{i}$ of $\Omega$ ) is given by

(*) $y \in P_{i}(x)$ if and only if

$$
v_{i}(y) \equiv \sum_{s \in \Omega} \pi_{i}(s) u_{i}(s, y(s))>\sum_{s \in \Omega} \pi_{i}(s) u_{i}(s, x(s)) \equiv v_{i}(x), \quad x, y \in X_{i} .
$$

Suppose there is a Hausdorff vector space topology $\eta$ on $E$ such that all order intervals in $E$ are $\eta$-compact, and suppose that for each $i \in I$ : 
(a) $u_{i}(s, \cdot)$ is lower semi-continuous for all $s \in \Omega$ (for the original topology of $E$ ).

(b) $u_{i}(s, \cdot)$ is $\eta$-upper semi-continuous for all $s \in \Omega$.

(c) $u_{i}(s, \cdot)$ is concave for all $s \in \Omega$.

(d) There is a $z_{i} \in E_{+}$and for each $s \in \Omega$ a convex open cone $\Gamma_{i, s} \subset E$ with $z_{i} \in \Gamma_{i, s}$ so that $e \in E_{+}, \gamma \in \Gamma_{i . s}$, and $e+\gamma \in E_{+}$imply $u_{i}(s, e+\gamma)>u_{i}(s, e)$.

Thus (d) means that the preferences given by $u_{i}(s, \cdot)$ are uniformly proper for each state $s \in \Omega$ and, moreover, that there is a $z_{i} \in E_{+}$that is a strongly desirable consumption bundle in each single state $s \in \Omega$.

It is readily seen that under these stated conditions, assumptions (A1) to (A5) and (A7) hold. (For (A7), pick any $i \in I$ and set $\Gamma_{i}=\prod_{s \in \Omega} \Gamma_{i, s}$. Then from (d):

$(* *) \quad$ If $y \in X_{i}$ and $\gamma \in \Gamma_{i}$ satisfy $y+\gamma \in X_{i}$ then $y+\gamma \in P_{i}(y)$.

Fix any $x \in X_{i}$ and let $\widetilde{P}_{i}(x)=P_{i}(x)+\left(\Gamma_{i} \cup\{0\}\right)$. From $(*), \widetilde{P}_{i}(x) \cap X_{i}=P_{i}(x)$, because $P_{i}$, coming from a utility function, is transitive. Clearly $\widetilde{P}_{i}(x)$ is convex. Further $\Gamma_{i}$ is open in $E^{\Omega}$, and hence so is $P_{i}(x)+\Gamma_{i}$. Thus $P_{i}(x)+\Gamma_{i} \subset \operatorname{int} \widetilde{P}_{i}(x)$. Consider the element $z_{i}$ from (d), and let $\bar{\gamma}_{i} \in E^{\Omega}$ be defined by $\bar{\gamma}_{i}(s)=z_{i}$ for each $s \in \Omega$. Then $\bar{\gamma}_{i} \in \Gamma_{i}$ and, being constant across states, $\bar{\gamma}_{i} \in X_{i}$. It follows that $x+\bar{\gamma}_{i} \in X_{i}$ and hence from $(* *)$ that $x+\bar{\gamma}_{i} \in P_{i}(x)$. Consequently $x+2 \bar{\gamma}_{i} \in P_{i}(x)+\Gamma_{i}$ as well as $x+2 \bar{\gamma}_{i} \in X_{i}$. Thus (int $\left.\widetilde{P}_{i}(x)\right) \cap X_{i} \neq \varnothing$. We conclude that for each $i \in I, P_{i}$ is ATY-proper at each $x \in X_{i}$. In particular, (A7) holds. As for (A3) and (A4), simply note that for each $x \in X_{i}$ and each real number $\lambda>0, x+\lambda \bar{\gamma}_{i} \in P_{i}(x)$. Of course, there are conditions on the utility functions $u_{i}$ so that (A3) and (A4) would hold independently of a properness assumption.)

In the following, the term feasible allocation means, as in the previous section, physically feasible without free disposal as well as informationally feasible, i.e for each agent $i \in I$ the consumption bundle must be in the informationally constrained consumption set $X_{i}$.

Definition 2. A Walrasian expectations equilibrium (WEE) for the economy $\mathcal{E}$ is a pair $\left(\left(x_{i}\right)_{i \in I}, p\right)$ where $\left(x_{i}\right)_{i \in I}$ is a feasible allocation and $p \in E^{\Omega \text {,* }}$ is a price system such that for each $i \in I$,

(i) $p x_{i} \leq p w_{i}$ and

(ii) $p y>p w_{i}$ whenever $y \in X_{i}$ satisfies $v_{i}(y)>v_{i}(x)$.

In view of $(*)$ and the discussion following $(*)$, Theorems 1-3 (together with some irreducibility assumption on the economy) entail existence results for Walrasian expectations equilibria. Notice that such results extend the ones in Aliprantis, Tourky, and Yannelis (2001) who allowed for free disposal in the definition of the Walrasian expectations equilibrium. 
As pointed out in the introduction, when the definition of feasibility of allocations allows for free disposal, then an equilibrium of a differential information economy may lack incentive compatibility properties. Indeed, consider the following definition of incentive compatibility due to Krasa and Yannelis (1994), where given $i \in I$ and $s \in \Omega, S_{i}(s) \in \mathcal{H}_{i}$ denotes that element of agent $i$ 's information partition which contains the state $s$.

Definition 3. A feasible (in particular informationally feasible) allocation $\left(x_{i}\right)_{i \in I}$ is said to be coalitional incentive compatible (CIC) if the following is not true: There are a coalition $C \subset I$ and states $s, s^{\prime} \in \Omega$ with $s \neq s^{\prime}$ such that $s^{\prime} \in S_{i}(s)$ for each $i \in I \backslash C$ and such that for each $i \in C, w_{i}(s)+x_{i}\left(s^{\prime}\right)-w_{i}\left(s^{\prime}\right) \geq 0$ and satisfies $u_{i}\left(s, w_{i}(s)+x_{i}\left(s^{\prime}\right)-w_{i}\left(s^{\prime}\right)\right)>u_{i}\left(s, x_{i}(s)\right){ }^{4}$

This definition indicates that no coalition $C$ of agents can misreport a realized (true) state of nature to the complementary coalition (whose members cannot distinguish the true state from the misreported one) and become better off. For example, suppose $I=\{1,2\}, \Omega=\{a, b, c\}$, and $E=\mathbb{R}$, i.e. there is one physical good. Let probability assignments, information sets, and endowments be given as follows:

$$
\begin{array}{lll}
\pi_{1}=(1 / 3,1 / 3,1 / 3) & \mathcal{H}_{1}=\{\{a, b\},\{c\}\} & w_{1}=(9,9,1) \\
\pi_{2}=(1 / 3,1 / 3,1 / 3) & \mathcal{H}_{2}=\{\{a, c\},\{b\}\} & w_{2}=(9,1,9) .
\end{array}
$$

Finally, let utility functions $u_{1}: \Omega \times \mathbb{R}_{+} \rightarrow \mathbb{R}$ and $u_{2}: \Omega \times \mathbb{R}_{+} \rightarrow \mathbb{R}$ be given by

$$
u_{1}(s, y)=u_{2}(s, y)=y^{1 / 2} \text { for all } s \in \Omega \text { and all } y \in \mathbb{R} \text {. }
$$

In this example, there is a unique free disposal WEE allocation given by

$$
x_{1}=(8,8,2) \quad x_{2}=(8,2,8) .
$$

(A price system supporting this allocation as a free disposal WEE is given by $p=(0,1,1)$.) Note that this allocation is not CIC. Indeed, when $a$ is the realized state of nature, agent 2 can report state $b$ (note agent 1 cannot distinguish $a$ from $b$ ) and becomes better off, i.e. keep the initial endowment in state $a$, which is 9 , and add the unit of the good she receives in state $b$ from agent 1 , thus realizing a utility

$$
u_{2}\left(a, w_{2}(a)+x_{2}(b)-w_{2}(b)\right)=u_{2}(a, 10)>u_{2}(a, 8)=u_{2}\left(a, x_{2}(a)\right) .
$$

On the other hand, it may be seen that for the price system $p=(-1 / 3,1,1)$ the initial allocation $\left(w_{1}, w_{2}\right)$ is a non-free disposal WEE allocation, and this allocation is evidently CIC. ${ }^{5}$ We remark that it is shown in Hervés-Beloso, MorenoGarcía, and Yannelis (2005, Theorem 5.1) that, in fact, any non-free disposal

\footnotetext{
${ }^{4}$ There is another, stronger definition of incentive compatibility in Krasa and Yannelis (1994); what we have defined here is actually called weak coalitional incentive compatible in Krasa and Yannelis (1994).

${ }^{5}$ The initial allocation is the only non-free disposal WEE allocation in this example. However, it is easy to construct examples-with more than three states-such that there are free-disposal WEE allocations that are not CIC, but non-free disposal WEE that involve some trading.
} 
WEE allocation is CIC, provided that utility functions satisfy some monotonicity and continuity properties. ${ }^{6}$ We take this discussion as ample justification for not going the convenient way of a free-disposal equilibrium in this paper.

We finish this section with a few remarks regarding the private core of a differential information economy.

Definition 4. Given an economy $\mathcal{E}=\left[\left(\mathcal{H}_{i}, P_{i}, w_{i}\right)_{i \in I}\right]$, an allocation $\left(x_{i}\right)_{i \in I}$ is said to be in the private core of the economy $\mathcal{E}$ if it is feasible and if there does not exist a (non-empty) coalition $C \subset I$ and an allocation $\left(y_{i}\right)_{i \in I}$ such that both $y_{i} \in P_{i}\left(x_{i}\right)$ for all $i \in C$ and $\sum_{i \in C} y_{i}=\sum_{i \in C} w_{i}$.

Recall that according to our terminology, the term "allocation" involves the requirement that the individual consumption bundles be informationally feasible. Thus the private information constraints are inherent in the above core notion. Clearly, the private core is just the usual core relative to the informationally constrained consumption sets. Thus, by a standard fact, any Walrasian equilibrium of a differential information economy belongs to the private core. (In particular, for the expected utility framework, any Walrasian expectations equilibrium allocation belongs to the private core.) Consequently, Theorems 1-3 imply results about non-emptiness of the private core of a differential information economy with an infinite dimensional commodity space. Note that Theorems 1-3 do not require the commodity space to be a Banach lattice. Thus, such private core existence results do not follow from any other core existence results which require the commodity space to be a Banach lattice with an order continuous norm (e.g. Allen, 2005; Lefebvre, 2001; Page, 1997; Yannelis, 1991).

The fact that a Walrasian equilibrium allocation of an asymmetric information economy belongs to the private core means in particular that any such allocation is Pareto optimal relative to the given information constraints. On the other hand, returning to the expected utility framework, it is important to notice that it is not possible to have fully or unconstrained Pareto optimal allocations which are also incentive compatible. ${ }^{7}$ (See for example Glycopantis and Yannelis, 2005a, p. vi. ) However, by the remarks above, a Walrasian expectations equilibrium allocation is both incentive compatible (under some mild assumptions) and informationally constrained (or second best) Pareto optimal. This should be contrasted with the Rational expectations equilibrium (revealing or non-revealing) which may not exist, may not be incentive compatible, and may not be Pareto optimal. (See for example Glycopantis and Yannelis, 2005b, p. 27, p. 31-32, and p. 43-45.)

\footnotetext{
${ }^{6}$ Actually, Theorem 5.1 in Hervés-Beloso et al. (2005) is stated for the commodity space $E=\ell_{\infty}$, but this specification is not needed in the proof of that theorem.

${ }^{7}$ Under asymmetric information, incentive compatibility is neither consistent with ex ante, interim, nor ex-post efficiency.
} 


\section{Proofs}

\subsection{Lemmata}

Lemma 1. Let $\Gamma=\left(X_{i}, A_{i}, P_{i}\right)_{i \in I}$ be an abstract economy, where $I$ is a finite set of agents, and for each $i \in I, X_{i}$ is a subset of $\mathbb{R}^{\ell}$ and both $A_{i}: \prod_{j \in I} X_{j} \rightarrow 2^{X_{i}}$ and $P_{i}: \prod_{j \in I} X_{j} \rightarrow 2^{X_{i}}$ are correspondences such that for each $i \in I$ :

(B1) $X_{i}$ is a non-empty, compact, and convex subset of $\mathbb{R}^{\ell}$.

(B2) $P_{i}$ is lower semi-continuous and has (relatively) open upper sections.

(B3) $A_{i}$ is non-empty and convex valued and has a (relatively) open graph in $\prod_{j \in I} X_{j} \times X_{i}$.

(B4) The correspondence $\bar{A}_{i}: \prod_{j \in I} X_{j} \rightarrow 2^{X}$, defined by $\bar{A}_{i}(x)=\mathrm{c} \ell A_{i}(x)$, is upper semi-continuous.

(B5) For all $x \in \prod_{j \in I} X_{i}, x_{i}$ does not belong to the convex hull of $P_{i}(x)$.

Then $\Gamma$ has an equilibrium, i.e. there is an $x^{*} \in \prod_{i \in I} X_{i}$ such that for all $i \in I$,

(i) $x_{i}^{*} \in \mathrm{c} \ell A_{i}\left(x^{*}\right)$, and

(ii) $P_{i}\left(x^{*}\right) \cap \mathrm{c} \ell A_{i}\left(x^{*}\right)=\varnothing$.

Proof. See Theorem A.1 in Won and Yannelis (2005).

Lemma 2. Let $\mathcal{E}=\left[\left(X_{i}, P_{i}, w_{i}\right)_{i \in I}\right]$ be an economy with commodity space $\mathbb{R}^{\ell}$ and with a finite set I of agents where for each $i \in I, X_{i}$ is the consumption set, $w_{i}$ is the initial endowment, and $P_{i}: X_{i} \rightarrow 2^{X_{i}}$ is the preference relation. Suppose the following conditions.

(C1) $X_{i}$ is closed and convex for each $i \in I$.

(C2) There is a closed convex cone $\Lambda \subset \mathbb{R}^{\ell}$, satisfying $\Lambda \cap-\Lambda=\{0\}$, such that $X_{i} \subset \Lambda$ for each $i \in I$.

(C3) $w_{i} \in X_{i}$ for each $i \in I$.

(C4) For each $i \in I, P_{i}: X_{i} \rightarrow 2^{X_{i}}$ has a (relatively) open graph in $X_{i} \times X_{i}$.

(C5) For each $i \in I$ and each $x_{i} \in X_{i}, P_{i}\left(x_{i}\right)$ is convex and $x_{i} \notin P_{i}\left(x_{i}\right)$.

(C6) If $\left(x_{i}\right)_{i \in I}$ is a feasible and individually rational allocation then $P_{i}\left(x_{i}\right) \neq \varnothing$.

Then $\mathcal{E}$ has a quasi-equilibrium $\left(\left(x_{i}^{*}\right)_{i \in I}, p^{*}\right)$-in particular, $p^{*} \neq 0$-such that the allocation $\left(x_{i}^{*}\right)_{i \in I}$ is individually rational. ${ }^{8}$

\footnotetext{
${ }^{8}$ We do not claim, of course, that this quasi-equilibrium is non-trivial.
} 
Proof. ${ }^{9}$ Since preferences are not assumed to be locally non-satiated, we will make use of the following construction, which goes back to Gale and Mas-Colell (1975). For each $i \in I$ define a correspondence $\widetilde{P}_{i}: X_{i} \rightarrow 2^{X_{i}}$ by

$$
\widetilde{P}_{i}\left(x_{i}\right)=\left\{(1-\alpha) x_{i}+\alpha x_{i}^{\prime}: 0<\alpha \leq 1, x_{i}^{\prime} \in P_{i}\left(x_{i}\right)\right\}, x_{i} \in X_{i} .
$$

Then $\widetilde{P}_{i}\left(x_{i}\right) \neq \varnothing$ if and only if $P_{i}\left(x_{i}\right) \neq \varnothing$. In particular, by (C6), if $\left(x_{i}\right)_{i \in I}$ is a feasible and individually rational allocation, then $x_{i} \in \mathrm{c} \ell \widetilde{P}_{i}\left(x_{i}\right)$ for each $i \in I$. Evidently, by (C5), $x_{i} \notin \widetilde{P}_{i}\left(x_{i}\right)$ and $\widetilde{P}_{i}\left(x_{i}\right)$ is convex for all $x_{i} \in X_{i}$. Finally, note that (C4) implies that each $P_{i}$ is lower semi-continuous and has relatively open upper sections. Hence, by Proposition 2.3.1 of Won and Yannelis (2005), the same is true for each $\widetilde{P}_{i}$.

For each $i \in I$, let $X_{i}^{f}$ be the set of those $x_{i} \in X_{i}$ that belong to feasible allocations. By (C1) and (C2), $X_{i}^{f}$ is compact for each $i \in I$. Choose and fix a compact and convex subset $K$ of $\mathbb{R}^{\ell}$ such that for each $i \in I, X_{i}^{f} \subset \operatorname{int} K$ and let $\hat{X}_{i}=X_{i} \cap K$. Note that by (C1), $\hat{X}_{i}$ is compact and convex for each $i \in I$. Also note that $w_{i} \in \hat{X}_{i} \cap \operatorname{int} K$ for each $i \in I$, since $w_{i} \in X_{i}$ according to (C3) and since the allocation $\left(w_{i}\right)_{i \in I}$ is feasible.

Write $I=\{1, \ldots, m\}$ and let $I^{\prime}=\{0\} \cup I$. Set $\hat{X}_{0}=\left\{p \in \mathbb{R}^{\ell}:\|p\| \leq 1\right\}$ and let $\hat{X}=\prod_{i \in I^{\prime}} \hat{X}_{i}$. We will denote elements of $\hat{X}$ by $(p, x)$ where $p \in \hat{X}_{0}$ and $x=\left(x_{1}, \ldots, x_{m}\right) \in \prod_{i=1}^{m} \hat{X}_{i}$. For each $i \in I$ define a correspondence $\hat{P}_{i}: \hat{X} \rightarrow 2^{\hat{X}_{i}}$ by

$$
\hat{P}_{i}(p, x)=\widetilde{P}_{i}\left(x_{i}\right) \cap \operatorname{int} K,
$$

and let $\hat{P}_{0}: \hat{X} \rightarrow 2^{\hat{X}_{0}}$ be defined by

$$
\hat{P}_{0}(p, x)=\left\{q \in \hat{X}_{0}: q \sum_{i \in I}\left(x_{i}-w_{i}\right)>p \sum_{i \in I}\left(x_{i}-w_{i}\right)\right\} .
$$

Evidently $\hat{P}_{0}$ has relatively open lower and relatively open upper sections. The fact that $\hat{P}_{0}$ has relatively open lower sections implies that $\hat{P}_{0}$ is lower semicontinuous. As noted above, for each $i \in I$ the correspondence $\widetilde{P}_{i}$ is lower semicontinuous and has relatively open upper sections. Hence, for each $i \in I$, the same is true for $\hat{P}_{i}$. (To see that $\hat{P}_{i}$ is lower semi-continuous for $i \in I$, let $O$ be a relatively open subset of $\hat{X}_{i}$. Then $O=U \cap \hat{X}_{i}$ where $U$ is a relatively open subset of $X_{i}$. Observe that

$$
\begin{aligned}
\left\{(p, x) \in \hat{X}: \hat{P}_{i}(p, x) \cap O \neq \varnothing\right\} & \\
& =\left\{(p, x) \in \hat{X}:\left(\widetilde{P}_{i}\left(x_{i}\right) \cap \operatorname{int} K\right) \cap O \neq \varnothing\right\} \\
& =\left\{(p, x) \in \hat{X}: \widetilde{P}_{i}\left(x_{i}\right) \cap\left(X_{i} \cap \operatorname{int} K\right) \cap U \neq \varnothing\right\} .
\end{aligned}
$$

But since $\widetilde{P}_{i}$ is lower semi-continuous and both $U$ and $X_{i} \cap \operatorname{int} K$ are relatively open in $X_{i}$, the set $\left\{x_{i} \in X_{i}: \widetilde{P}_{i}\left(x_{i} \cap\left(X_{i} \cap \operatorname{int} K\right) \cap U\right) \neq \varnothing\right\}$ is relatively open

\footnotetext{
${ }^{9}$ The proof follows that of Theorem 2.3.1 in Won and Yannelis (2005). However, some additional constructions become necessary because we have not assumed that the endowments be interior points of the consumption sets.
} 
in $X_{i}$, whence the set $\left\{(p, x) \in \hat{X}: \widetilde{P}_{i}\left(x_{i}\right) \cap\left(X_{i} \cap\right.\right.$ int $\left.\left.K\right) \cap U \neq \varnothing\right\}$ is relatively open in $\hat{X}$. Thus $\hat{P}_{i}$ is lower semi-continuous.)

Next, for each $i \in I$, and each integer $k>0$, let a correspondence $A_{i}^{k}: \hat{X} \rightarrow 2^{\hat{X}_{i}}$ be defined by

$$
A_{i}^{k}(p, x)=\left\{x_{i}^{\prime} \in \hat{X}_{i}: p x_{i}^{\prime}<p w_{i}+(1 / k)+1-\|p\|\right\} .
$$

Then each $A_{i}^{k}$ is non-empty valued because $w_{i} \in \hat{X}_{i}$ for each $i \in I$. Also, each $A_{i}^{k}$ is convex valued, has a relatively open graph in $\hat{X} \times \hat{X}_{i}$, and the correspondences $\bar{A}_{i}^{k}: \hat{X} \rightarrow 2^{\hat{X}_{i}}$, defined by $\bar{A}_{i}^{k}(p, x)=\mathrm{c} \ell A_{i}^{k}(p, x)$, are upper semi-continuous.

Finally, for each integer $k>0$, define a correspondence $A_{0}^{k}: \hat{X} \rightarrow 2^{\hat{X}_{0}}$ by

$$
A_{0}^{k}(p, x)=\hat{X}_{0} .
$$

Clearly, for each $k, A_{0}^{k}$ is non-empty and convex valued, has a relatively open graph in $\hat{X} \times \hat{X}_{0}$, and is upper semi-continuous. Observe also that for each $(p, x) \in \hat{X}$ we have $c \ell A_{0}^{k}(p, x)=A_{0}^{k}(p, x)$.

For each integer $k>0$ consider the abstract economy $\Gamma^{k}=\left(\hat{X}_{i}, A_{i}^{k}, \hat{P}_{i}\right)_{i \in I^{\prime}}$. By the remarks above, each $\Gamma^{k}$ satisfied the hypotheses of Lemma 1 . Thus each $\Gamma^{k}$ has an equilibrium, i.e. there is a $\left(p^{k}, x^{k}\right) \in \hat{X}$ such that

$$
p^{k} \sum_{i \in I}\left(x_{i}^{k}-w_{i}\right) \geq q \sum_{i \in I}\left(x_{i}^{k}-w_{i}\right) \text { for all } q \in \hat{X}_{0},
$$

and such that for each $i \in I$,

$$
\begin{gathered}
p^{k} x_{i}^{k} \leq p^{k} w_{i}+(1 / k)+1-\left\|p^{k}\right\|, \text { and } \\
\hat{P}_{i}\left(p^{k}, x^{k}\right) \cap\left\{x_{i} \in \hat{X}_{i}: p^{k} x_{i} \leq p^{k} w_{i}+(1 / k)+1-\left\|p^{k}\right\|\right\}=\varnothing .
\end{gathered}
$$

Since $w_{i} \in \hat{X}_{i}$ for each $i \in I$, (1c) implies in particular that $w_{i} \notin \hat{P}_{i}\left(p^{k}, x^{k}\right)$ for each $i \in I$. Hence, by the construction of $\hat{P}_{i}$, since $w_{i} \in \operatorname{int} K$ as noted above,

$$
w_{i} \notin P_{i}\left(x_{i}^{k}\right) \text { for each } i \in I .
$$

The set $\hat{X}$ is compact. Therefore, letting $k \rightarrow \infty$ and passing to a subsequence if necessary, we can assume that there is a $\left(p^{*}, x^{*}\right) \in \hat{X}$ such that $p^{k} \rightarrow p^{*}$ and $x_{i}^{k} \rightarrow x_{i}^{*}$ for each $i \in I$. Then from (1),

$$
\begin{gathered}
p^{*} \sum_{i \in I}\left(x_{i}^{*}-w_{i}\right) \geq q \sum_{i \in I}\left(x_{i}^{*}-w_{i}\right) \text { for all } q \in \hat{X}_{0}, \\
p^{*} x_{i}^{*} \leq p^{*} w_{i}+1-\left\|p^{*}\right\| \text { for each } i \in I,
\end{gathered}
$$

and, since $\hat{P}_{i}$ is lower semi-continuous for each $i \in I$,

$$
\hat{P}_{i}\left(p^{*}, x^{*}\right) \cap\left\{x_{i} \in \hat{X}_{i}: p^{*} x_{i}<p^{*} w_{i}+1-\left\|p^{*}\right\|\right\}=\varnothing \text { for each } i \in I .
$$


From (3a) and (3b) it is plain that $\sum_{i \in I} x_{i}^{*}=\sum_{i \in I} w_{i}$, i.e. the allocation $\left(x_{i}^{*}\right)_{i \in I}$ is feasible. From (2), $w_{i} \notin P_{i}\left(x_{i}^{*}\right)$ for each $i \in I$ since by (C4) each $P_{i}$ has relatively open lower sections. That is, the allocation $\left(x_{i}^{*}\right)_{i \in I}$ is individually rational. Pick any $j \in I$. By what has been remarked at the beginning of this proof, the fact that the allocation $\left(x_{i}^{*}\right)_{i \in I}$ is both feasible and individually rational implies that $x_{j}^{*} \in \mathrm{c} \ell \widetilde{P}_{j}\left(x_{j}^{*}\right)$. Also, again since $\left(x_{i}^{*}\right)_{i \in I}$ is feasible, we must have $x_{j}^{*} \in \operatorname{int} K$ by choice of $K$. Thus $x_{j}^{*} \in \mathrm{c} \ell\left(\widetilde{P}_{j}\left(x_{j}^{*}\right) \cap\right.$ int $\left.K\right)$. By definition of $\hat{P}_{j}$, this means $x_{j}^{*} \in \mathrm{c} \ell \hat{P}_{j}\left(p^{*}, x^{*}\right)$. Hence, by (3b) and (3c), $p^{*} x_{j}^{*}=p^{*} w_{j}+1-\left\|p^{*}\right\|$. Since this holds for all $i \in I$, it follows that $\left\|p^{*}\right\|=1$ since $\sum_{i \in I} p^{*} x_{i}^{*}=\sum_{i \in I} p^{*} w_{i}$ by feasibility of $\left(x_{i}^{*}\right)_{i \in I}$. Now pick any $i \in I$ and suppose $x_{i} \in P_{i}\left(x_{i}^{*}\right)$. Since $x_{i}^{*} \in \operatorname{int} K$, we have $(1-\alpha) x_{i}^{*}+\alpha x_{i} \in \widetilde{P}_{i}\left(x_{i}^{*}\right) \cap \operatorname{int} K \equiv \hat{P}_{i}\left(p^{*}, x^{*}\right)$ for all sufficiently small real numbers $\alpha>0$. Therefore (3b) and (3c), together with the fact that $\left\|p^{*}\right\|=1$, imply that $p^{*} x_{i} \geq p^{*} w_{i}$. This completes the proof of the lemma.

Lemma 3. Let $Y$ be a real locally convex Hausdorff space and let $U$ and $V$ be convex subsets of $Y$ with $U$ open and such that $U \cap V \neq \varnothing$. Let $y \in V \cap \mathrm{c} \ell U$, let $q$ be a (not necessarily continuous) linear functional on $Y$ and suppose $q y \leq q y^{\prime}$ for all $y^{\prime} \in U \cap V$. Then there exist linear functionals $q_{1}$ and $q_{2}$ on $Y$ such that $q_{1}$ is continuous, $q_{1} y \leq q_{1} u$ for all $u \in U, q_{2} y \leq q_{2} v$ for all $v \in V$, and $q_{1}+q_{2}=q$.

For a proof see Podczeck (1996, Lemma 2).

Lemma 4. Let $(E,\|\cdot\|)$ be a normed Riesz space with $\|\cdot\|$ being an order unit norm. Suppose there exists a Hausdorff linear topology $\eta$ on $E$ such that all order intervals in $E$ are $\eta$-compact. Then $(E,\|\cdot\|)$ is a Banach lattice.

Proof. ${ }^{10}$ The hypothesis about order intervals implies that $E$ has the so called interpolation property. That is, whenever $\left(x_{n}\right)$ and $\left(y_{n}\right)$ are sequences in $E$ such that $x_{n} \leq y_{m}$ for all $m, n$, then there is a $u \in E$ such that $x_{n} \leq u \leq y_{m}$ for all $m, n$. (Indeed, given any such sequences, for each $n$ let $a_{n}=\sup \left\{x_{m}: m \leq n\right\}$ and $\left.b_{n}=\inf \left\{y_{m}: m \leq n\right)\right\}$. Then $a_{n} \leq b_{n}$ for all $n$ and the sequence of order intervals $\left[a_{n}, b_{n}\right]$ is nested. Hence the hypothesis implies that $\bigcap_{n}\left[a_{n}, b_{n}\right] \neq \varnothing$. Evidently, any element $u$ of this intersection has the property that $x_{n} \leq u \leq y_{m}$ for all $m, n$.) Now since $E$, being a normed Riesz space, is Archimedean, the fact that $E$ has the interpolation property implies that $E$ is uniformly complete. But a normed uniformly complete Riesz space whose norm is an order unit norm is actually a Banach lattice.

In the proofs of the next lemmata, we follow the convention that the sum of indexed vectors over an empty index set is zero.

\footnotetext{
${ }^{10}$ For the terminology and the facts used in this proof, see Meyer-Nieberg (1991, Definition 1.1.7, p. 7, Proposition 1.1.8, p. 7, and Proposition 1.2.13, p. 18).
} 
Lemma 5. Let $(T, \mathcal{T}, \mu)$ be a probability space, let $U=\{1, \ldots, m\}, V=\{1, \ldots, n\}$, and $K$ be non-empty finite sets, and let $f_{1}, \ldots, f_{m}$ and $g_{1}, \ldots, g_{n}$ be non-negative measurable real-valued functions on $T$. For each $k \in K$, let $U_{k}$ be a non-empty subset of $U$ and $V_{k}$ be a non-empty subset of $V$. Suppose the following conditions to hold:

(i) $\bigcup_{k \in K} U_{k}=U$.

(ii) $\bigcup_{k \in K} V_{k}=V$

(iii) There are real numbers $b$ and $c$ and, for each $\ell=1,2, \ldots$, a measurable subset $C_{\ell}$ of $T$ with $\mu\left(C_{\ell}\right)>0$ such that:

(a) $\sum_{i \in U_{k}} f_{i}(t)-\sum_{i \in V_{k}} g_{i}(t) \geq b$ for all $k \in K$ and all $t \in \bigcup_{\ell=1}^{\infty} C_{\ell}$.

(b) $g_{i}(t) \geq \ell$ for all $t \in C_{\ell}$, each $\ell$, and each $i \in V$.

(c) $c>0$ and $f_{i}(t) \geq c$ for all $t \in \bigcup_{\ell=1}^{\infty} C_{\ell}$ and each $i \in U$.

Then, given real numbers $r>0$ and $\epsilon>0$, there are non-negative measurable real-valued functions $f_{1}^{\prime}, \ldots, f_{m}^{\prime}$ and $g_{1}^{\prime}, \ldots, g_{n}^{\prime}$ on $T$ and a measurable subset $D$ of $T$, with $D \subset \bigcup_{\ell=1}^{\infty} C_{\ell}$ and $\mu(D)>0$, such that:

(I) For all $t \in D, f_{i}^{\prime}(t) \leq f_{i}(t)$ for each $i \in U$ and $g_{i}^{\prime}(t) \leq g_{i}(t)$ for each $i \in V$.

(II) For some $i \in U, f_{i}^{\prime}(t)<f_{i}(t)$ for all $t \in D$.

(III) For all $t \in T \backslash D, f_{i}^{\prime}(t)=f_{i}(t)$ for each $i \in U$ as well as $g_{i}^{\prime}(t)=g_{i}(t)$ for each $i \in V$.

(IV) $f_{i}^{\prime}(t) \geq f_{i}(t)-\epsilon$ for all $t \in T$ and all $i \in U$.

(V) For all $t \in T$ and all $k \in K$ :

$$
\sum_{i \in U_{k}} f_{i}^{\prime}(t)-\sum_{i \in V_{k}} g_{i}^{\prime}(t) \leq \sum_{i \in U_{k}} f_{i}(t)-\sum_{i \in V_{k}} g_{i}(t)
$$

and if $\sum_{i \in U_{k}} f_{i}(t)-\sum_{i \in V_{k}} g_{i}(t) \leq r$ then

$$
\sum_{i \in U_{k}} f_{i}^{\prime}(t)-\sum_{i \in V_{k}} g_{i}^{\prime}(t)=\sum_{i \in U_{k}} f_{i}(t)-\sum_{i \in V_{k}} g_{i}(t)
$$

Proof. Set $C=\bigcup_{\ell=1}^{\infty} C_{\ell}$, let $G$ be the set of all subsets of $K$, and let $r>0$ be a real number. For each $G \in G$ let

$$
\begin{aligned}
& C_{G}=\{t \in C: \sum_{i \in U_{k}} f_{i}(t)-\sum_{i \in V_{k}} g_{i}(t) \leq r \text { for all } k \in G \\
&\text { and } \left.\sum_{i \in U_{k}} f_{i}(t)-\sum_{i \in V_{k}} g_{i}(t)>r \text { for all } k \in K \backslash G\right\} .
\end{aligned}
$$


Then each $C_{G}$ is a measurable set and we have $C=\cup_{G \in G} C_{G}$. Thus since $K$ and hence $G$ is a finite set, at least one of the $G$ 's must have the property that $\mu\left(C_{G} \cap C_{\ell}\right)>0$ for infinitely many $\ell$ (because $\mu\left(C_{\ell}\right)>0$ for each $\ell$ ). Choose and fix any element of $G$ with this property, say $\bar{K}$, and let $\left(C_{j}\right)$ be the subsequence of the sequence $\left(C_{\ell}\right)$ such that $\mu\left(C_{\bar{K}} \cap C_{j}\right)>0$ holds for each $j=1,2, \ldots$. Set $D_{j}=C_{\bar{K}} \cap C_{j}$ for each $j$ and set $D=\bigcup_{j=1}^{\infty} D_{j}$. Thus,

$$
\text { if } t \in D \text { then } \sum_{i \in U_{k}} f_{i}(t)-\sum_{i \in V_{k}} g_{i}(t) \leq r \text { if and only if } k \in \bar{K}
$$

and, from (iiib),

(5) for each $j, \mu\left(D_{j}\right)>0$ and $g_{i}(t) \geq j$ for all $t \in D_{j}$ and each $i \in V$.

For each $j=1,2, \ldots$, pick a point $t^{j} \in D_{j}$ and set $\alpha^{j}=\sum_{i=1}^{m} f_{i}\left(t^{j}\right)$. Observe that by (5) and condition (iiia), $\alpha^{j} \rightarrow \infty$ as $j \rightarrow \infty$. In particular, $\alpha^{j}>0$ for all sufficiently large $j$. Thus we may as well assume that $\alpha^{j}>0$ holds for all $j$. Set $\alpha_{i}^{j}=\left(1 / \alpha^{j}\right) f_{i}\left(t^{j}\right)$ for each $i \in U$ and $\beta_{i}^{j}=\left(1 / \alpha^{j}\right) g_{i}\left(t^{j}\right)$ for each $i \in V$. Note that, for the number $b$ from condition (iii), we have

$$
\left(1 / \alpha^{j}\right) r \geq \sum_{i \in U_{k}} \alpha_{i}^{j}-\sum_{i \in V_{k}} \beta_{i}^{j} \geq\left(1 / \alpha^{j}\right) b \text { for all } k \in \bar{K}
$$

and

$$
\sum_{i \in U_{k}} \alpha_{i}^{j}-\sum_{i \in V_{k}} \beta_{i}^{j} \geq\left(1 / \alpha^{j}\right) b \text { for all } k \in K \backslash \bar{K}
$$

Also, we have $0 \leq \alpha_{i}^{j} \leq 1$ for each $j$ and each $i \in U$. That is, for each $i \in U$ the sequence $\left.\left(\alpha_{i}^{j}\right)\right)_{j=1,2, \ldots}$ is bounded. But therefore, by (6) and condition (ii), the sequences $\left.\left(\beta_{i}^{j}\right)\right)_{j=1,2, \ldots}$ are also bounded since all the $\beta_{i}^{j}$ are non-negative and since $\alpha^{j} \rightarrow \infty$. Thus (passing to subsequences and relabeling if necessary) we may assume that all these sequences of real numbers are convergent, say $\alpha_{i}^{j} \rightarrow \alpha_{i}$ for each $i \in U$ and $\beta_{i}^{j} \rightarrow \beta_{i}$ for each $i \in V$. Then $\alpha_{i} \geq 0$ for all $i \in U$ and $\beta_{i} \geq 0$ for all $i \in V$, and since $\sum_{i=1}^{m} \alpha_{i}^{j}=1$ for each $j$ by construction, we must have $\alpha_{i}>0$ for some $i \in U$. Moreover, since $\alpha^{j} \rightarrow \infty$, a glance at (6) shows that

$$
\sum_{i \in U_{k}} \alpha_{i}-\sum_{i \in V_{k}} \beta_{i}=0 \text { for all } k \in \bar{K}
$$

and

$$
\sum_{i \in U_{k}} \alpha_{i}-\sum_{i \in V_{k}} \beta_{i} \geq 0 \text { for all } k \in K \backslash \bar{K}
$$

Let $\gamma>0$ be any real number, and consider the measurable functions $\gamma \alpha_{i} 1_{D}$, $i \in U$, and $\gamma \beta_{i} 1_{D}, i \in V$. Then from (7),

$$
\sum_{i \in U_{k}} \gamma \alpha_{i} 1_{D}(t)-\sum_{i \in V_{k}} \gamma \beta_{i} 1_{D}(t)=0 \text { for all } t \in T \text { and all } k \in \bar{K}
$$


and

$$
\sum_{i \in U_{k}} \gamma \alpha_{i} 1_{D}(t)-\sum_{i \in V_{k}} \gamma \beta_{i} 1_{D}(t) \geq 0 \text { for all } t \in T \text { and all } k \in K \backslash \bar{K} \text {. }
$$

Hence, setting $f_{i}^{\prime}=f_{i}-\gamma \alpha_{i} 1_{D}$ for each $i \in U$ and $g_{i}^{\prime}=g_{i}-\gamma \beta_{i} 1_{D}$ for each $i \in V$, we obtain measurable functions on $T$ such that

$$
\sum_{i \in U_{k}} f_{i}^{\prime}(t)-\sum_{i \in V_{k}} g_{i}^{\prime}(t)=\sum_{i \in U_{k}} f_{i}(t)-\sum_{i \in V_{k}} g_{i}(t) \text { for all } t \in T \text { and all } k \in \bar{K}
$$

and

$$
\sum_{i \in U_{k}} f_{i}^{\prime}(t)-\sum_{i \in V_{k}} g_{i}^{\prime}(t) \leq \sum_{i \in U_{k}} f_{i}(t)-\sum_{i \in V_{k}} g_{i}(t) \text { for all } t \in T \text { and all } k \in K \backslash \bar{K} \text {. }
$$

Then the functions $f_{i}^{\prime}$ and $g_{i}^{\prime}$ satisfy (III) and, by (4), (V) of the required properties. Also, (I) and (II) hold since all the numbers $\alpha_{i}$ and $\beta_{i}$ are non-negative and since, as noted above, $\alpha_{i}>0$ for some $i \in U$ and $\mu(D)>0$. Furthermore, if $\gamma>0$ is chosen small enough, then by conditions (iiib) and (iiic) of the lemma, all these functions are non-negative. Finally, given any $\epsilon>0$, then again by choosing $\gamma>0$ sufficiently small we can guarantee that (IV) is satisfied, too.

Lemma 6. Let $(T, \mathcal{T}, \mu)$ be a probability space, let $f_{1}, \ldots, f_{n}$ and $g_{1}, \ldots, g_{n}$ be non-negative measurable real-valued functions on $T$, let $K$ be a non-empty finite set, and fore each $k \in K$ let $S_{k}$ be a non-empty subset of $\{1, \ldots, n\}$. Suppose the following conditions hold:

(i) $\bigcup_{k \in K} S_{k}=\{1, \ldots, n\}$.

(ii) $f_{i} \wedge g_{i}=0$ for each $i=1, \ldots, n$.

(iii) $\sum_{i \in S_{k}}\left(f_{i}-g_{i}\right) \geq-1_{T}$ for each $k \in K$.

(iv) There is no real number $\alpha>0$ such that for all $i=1, \ldots, n, g_{i}(t) \leq \alpha$ for almost all $t \in T$.

Then there are non-negative measurable real-valued functions $f_{1}^{\prime}, \ldots, f_{n}^{\prime}$ and $g_{1}^{\prime}, \ldots, g_{n}^{\prime}$ on $T$ such that:

(a) $f_{i}^{\prime} \leq f_{i}$ and $g_{i}^{\prime} \leq g_{i}$ for all $i=1, \ldots, n$.

(b) For some $i$ there is a set $D \in \mathcal{T}$ with $\mu(D)>0$ such that $f_{i}^{\prime}(t)<f_{i}(t)$ for all $t \in D$.

(c) $\sum_{i \in S_{k}}\left(f_{i}^{\prime}-g_{i}^{\prime}\right) \leq \sum_{i \in S_{k}}\left(f_{i}-g_{i}\right)$ for each $k \in K$.

(d) $\left(\sum_{i \in S_{k}}\left(f_{i}^{\prime}-g_{i}^{\prime}\right)\right) \wedge 1_{T}=\left(\sum_{i \in S_{k}}\left(f_{i}-g_{i}\right)\right) \wedge 1_{T}$ for each $k \in K$. 
Proof. Set $I=\{1, \ldots, n\}$ and let $V$ be a maximal subset of $I$ such that there is no real number $\alpha>0$ for which $\inf \left\{g_{i}(t): i \in V\right\} \leq \alpha$ for almost all $t \in T$ (where "maximal" is meant with respect to the number of elements). By condition (iv) of the lemma, $V$ is non-empty. Moreover, by the choice of $V$, we can find an integer $\bar{\ell}>0$ and measurable subsets $A_{\ell}$ of $T$ with $\mu\left(A_{\ell}\right)>0, \ell=1,2, \ldots$, such that for each $i \in V$ and each $\ell, g_{i}(t) \geq \ell$ for all $t \in A_{\ell}$ but for each $i \in I \backslash V, g_{i}(t) \leq \bar{\ell}$ for all $t \in \bigcup_{\ell=1}^{\infty} A_{\ell}$.

Set $A=\bigcup_{\ell=1}^{\infty} A_{\ell}$, let $\mathcal{F}$ be the set of all subsets of $I$, and for each $F \in \mathcal{F}$ let $A_{F}=\left\{t \in A: f_{i}(t)>n \bar{\ell}+3\right.$ for all $i \in F$ and $f_{i}(t) \leq n \bar{\ell}+3$ for all $\left.i \in I \backslash F\right\}$.

Then each $A_{F}$ is a measurable set and we have $A=\bigcup_{F \in \mathcal{F}} A_{F}$. Thus since $I$ and therefore $\mathcal{F}$ is a finite set, at least one of the $F$ 's must have the property that $\mu\left(A_{F} \cap A_{\ell}\right)>0$ for infinitely many $\ell$. Choose and fix any element of $\mathcal{F}$ with this property, say $W$, and let $\left(A_{\ell_{j}}\right)$ be the subsequence of the sequence $\left(A_{\ell}\right)$ such that $\mu\left(A_{W} \cap A_{\ell_{j}}\right)>0$ holds for each $j=1,2, \ldots$. For simplicity of notation, we denote this subsequence again by $\left(A_{\ell}\right)$. Set $C_{\ell}=A_{\ell} \cap A_{W}$ for each $\ell$, and $C=\bigcup_{\ell=1}^{\infty} C_{\ell}$.

Summarizing, we have subsets $V$ and $W$ of $I=\{1, \ldots, n\}$, with $V$ non-empty, a measurable subset $C$ of $T$, and a real number $\bar{\ell}>0$ such that:

(a) $C=\bigcup_{\ell=1}^{\infty} C_{\ell}$ where for each $\ell, C_{\ell} \in \mathcal{T}$ with $\mu\left(C_{\ell}\right)>0$ and $g_{i}(t) \geq \ell$ for all $t \in C_{\ell}$ and each $i \in V$.

(8) (b) For each $i \in I \backslash V, g_{i}(t) \leq \bar{\ell}$ for all $t \in C$.

(c) If $i \in W$ then $f_{i}(t)>n \bar{\ell}+3$ for all $t \in C$, and if $i \in I \backslash W$ then $f_{i}(t) \leq n \bar{\ell}+3$ for all $t \in C$.

Let

$$
\bar{K}=\left\{k \in K: S_{k} \cap V \neq \varnothing\right\} \quad \text { and } \quad U=\bigcup_{k \in \bar{K}}\left(S_{k} \cap W\right) .
$$

Then by condition (i) of the lemma, $\bar{K}$ is non-empty (since $V$ is non-empty) and we have $\bigcup_{k \in \bar{K}}\left(S_{k} \cap V\right)=V$. From (8a), (8c), and (iii) of the lemma, if $k \in \bar{K}$ then $S_{k} \cap W \neq \varnothing$. Hence, $U$ is non-empty, too. Note for later reference that $U \subset W$.

Now according to condition (iii) of the lemma, for each $k \in K$ and all $t \in T$

$$
\sum_{i \in S_{k}} f_{i}(t)-\sum_{i \in S_{k}} g_{i}(t) \geq-1
$$

and thus

$$
\sum_{i \in S_{k} \cap W} f_{i}(t)-\sum_{i \in S_{k} \cap V} g_{i}(t) \geq-1-\sum_{i \in S_{k} \cap(\Lambda W)} f_{i}(t)
$$

because all the functions involved are non-negative. By (8c), then, for each $k \in K$ (in particular for each $k \in \bar{K}$ ) and all $t \in C$,

$$
\sum_{i \in S_{k} \cap W} f_{i}(t)-\sum_{i \in S_{k} \cap V} g_{i}(t) \geq-1-n(n \ell+3) .
$$


In view of this, (8a), and the first part of (8c) together with the fact that $U \subset W$, we may now appeal to Lemma 5 , with $K$ there replaced by $\bar{K}, U_{k}$ by $S_{k} \cap W, V_{k}$ by $S_{k} \cap V$, and with $\epsilon=1 / n$ and $r=n \ell+2$, to obtain a measurable set $D \subset C$, with $\mu(D)>0$, and non-negative measurable functions $f_{i}^{\prime}: T \rightarrow \mathbb{R}, i \in U$, and $g_{i}^{\prime}: T \rightarrow \mathbb{R}, i \in V$, such that (I) to (V) of that lemma hold (with $\bar{K}$ in place of $K$, $S_{k} \cap W$ in place of $U_{k}$, and $S_{k} \cap V$ in place of $V_{k}$ ).

Set $f_{i}^{\prime}=f_{i}$ for $i \in I \backslash U$ and $g_{i}^{\prime}=g_{i}$ for $i \in I \backslash V$. Then, by (I) and (II) of Lemma 5, the functions $f_{i}^{\prime}$ and $g_{i}^{\prime}, i \in I$, evidently satisfy (a) and (b) of the properties required in the lemma just under proof. Note that for each $k \in K$ and each $t \in T$, we may write

$$
\sum_{i \in S_{k}} f_{i}^{\prime}(t)-\sum_{i \in S_{k}} g_{i}^{\prime}(t)=\sum_{i \in S_{k} \cap W} f_{i}^{\prime}(t)-\sum_{i \in S_{k} \cap V} g_{i}^{\prime}(t)+\sum_{i \in S_{k} \cap(I \cap W)} f_{i}^{\prime}(t)-\sum_{i \in S_{k} \cap(I \cap V)} g_{i}(t) .
$$

But from this it is plain that (c) must hold because we have $f_{i}^{\prime}(t) \leq f_{i}(t)$ for each $i \in I$, and because if $k \in \bar{K}$, i.e. $S_{k} \cap V \neq \varnothing$, then according to (V) of Lemma $5^{11}$

$$
\sum_{i \in S_{k} \cap W} f_{i}^{\prime}(t)-\sum_{i \in S_{k} \cap V} g_{i}^{\prime}(t) \leq \sum_{i \in S_{k} \cap W} f_{i}(t)-\sum_{i \in S_{k} \cap V} g_{i}(t)
$$

for all $t \in T$. Now as for (d) of the required properties, by (III) of Lemma 5 we have only to check that, for each $k \in K$, if $t$ is any point of $D$, then

$$
\left(\sum_{i \in S_{k}}\left(f_{i}^{\prime}(t)-g_{i}^{\prime}(t)\right)\right) \wedge 1=\left(\sum_{i \in S_{k}}\left(f_{i}(t)-g_{i}(t)\right)\right) \wedge 1 .
$$

Thus pick any $t \in D$. Clearly, if $k \notin \bar{K}$, i.e. $S_{k} \cap V=\varnothing$, and if in addition $S_{k} \cap U=\varnothing$, then (9) holds because in this case $f_{i}^{\prime}=f_{i}$ as well as $g_{i}^{\prime}=g_{i}$ for all $i \in S_{k}$. Suppose $S_{k} \cap V=\varnothing$ but $S_{k} \cap U \neq \varnothing$. Then from (8b) and (8c)

$$
\sum_{i \in S_{k}} f_{i}(t)-\sum_{i \in S_{k}} g_{i}(t)>n \bar{\ell}+3-n \bar{\ell}=3,
$$

recalling that $D \subset C$, that $U \subset W$, and that $f_{i}(t) \geq 0$ for all $i$. On the other hand, from (IV) of Lemma 5 , since $\epsilon$ has been specified as $\epsilon=1 / n$,

$$
\sum_{i \in S_{k}} f_{i}^{\prime}(t) \geq \sum_{i \in S_{k}} f_{i}(t)-1
$$

(because $f_{i}^{\prime}=f_{i}$ for $i \notin U$ ). Consequently, since $g_{i}^{\prime}=g_{i}$ for $i \in I \backslash V$ and $S_{k} \cap V=\varnothing$, we must have $\sum_{i \in S_{k}} f_{i}^{\prime}(t)-\sum_{i \in S_{k}} g_{i}^{\prime}(t) \geq 2$. Thus (9) holds. Now suppose $k \in \bar{K}$. Recall that we have specified the number $r$ from Lemma 5 as $r=n \ell+2$. Thus from (V) of that lemma, if $\sum_{i \in S_{k} \cap W} f_{i}(t)-\sum_{i \in S_{k} \cap V} g_{i}(t) \leq n \ell+2$ then

$$
\sum_{i \in S_{k} \cap W} f_{i}^{\prime}(t)-\sum_{i \in S_{k} \cap V} g_{i}^{\prime}(t)=\sum_{i \in S_{k} \cap W} f_{i}(t)-\sum_{i \in S_{k} \cap V} g_{i}(t)
$$

\footnotetext{
${ }^{11}$ Here and in the rest of this proof, all invocations of Lemma 5 are understood with $K$ replaced by $\bar{K}, U_{k}$ by $S_{k} \cap W$, and $V_{k}$ by $S_{k} \cap V$.
} 
and therefore, because $g_{i}^{\prime}=g_{i}$ for $i \in I \backslash V$ and because $I \backslash W \subset I \backslash U$ and $f_{i}^{\prime}=f_{i}$ for $i \in I \backslash U$,

$$
\sum_{i \in S_{k}} f_{i}^{\prime}(t)-\sum_{i \in S_{k}} g_{i}^{\prime}(t)=\sum_{i \in S_{k}} f_{i}(t)-\sum_{i \in S_{k}} g_{i}(t)
$$

Hence (9) holds again. Suppose $\sum_{i \in S_{k} \cap W} f_{i}(t)-\sum_{i \in S_{k} \cap V} g_{i}(t)>n \ell+2$. As noted above, we have $\sum_{i \in S_{k}} f_{i}^{\prime}(t) \geq \sum_{i \in S_{k}} f_{i}(t)-1$. Consequently, using the facts that $g_{i}^{\prime} \leq g_{i}$ for all $i$ and that all the $f_{i}$ 's are non-negative,

$$
\begin{aligned}
\sum_{i \in S_{k}} f_{i}^{\prime}(t)-\sum_{i \in S_{k}} g_{i}^{\prime}(t) & \geq \sum_{i \in S_{k}} f_{i}(t)-1-\sum_{i \in S_{k}} g_{i}^{\prime}(t) \\
& \geq \sum_{i \in S_{k}} f_{i}(t)-1-\sum_{i \in S_{k}} g_{i}(t) \\
& >n \ell+2-1-\sum_{i \in S_{k} \cap(I V V)} g_{i}(t) \\
& \geq 1,
\end{aligned}
$$

the last inequality following from (8b) (and since $t \in D \subset C$ ). Thus (9) follows in this latter case, too. Thus we have shown that also (d) holds for the functions $f_{i}^{\prime}$ and $g_{i}^{\prime}, i \in I$. This completes the proof of the lemma.

Lemma 7. Let $(T, \mathcal{T}, \mu)$ be a probability space, let $J$ and $K$ be non-empty finite sets, let $\left(S_{k}\right)_{k \in K}$ be a family of non-empty subset of $J$, and let $g_{j} \in L_{1}(\mu)$ for each $j \in J$. Suppose that $\sum_{j \in S_{k}} g_{j} \geq-1_{T}$ for each $k \in K$ and that $\bigcup_{k \in K} S_{k}=J$. Then there are an $h_{j} \in L_{1}(\mu)$ for each $j \in J$ and a real number $\alpha>0$ such that

(i) $h_{j} \geq-\alpha 1_{T}$ for each $j \in J$;

(ii) $\sum_{j \in S_{k}} h_{j} \leq \sum_{j \in S_{k}} g_{j}$ for each $k \in K$;

(iii) $\left(\sum_{j \in S_{k}} h_{j}\right) \wedge 1_{T}=\left(\sum_{j \in S_{k}} g_{j}\right) \wedge 1_{T}$ for each $k \in K$.

Proof. We regard $L_{1}(\mu)$ as endowed with the usual norm $\|\cdot\|_{1}$; thus $L_{1}(\mu)$ is a Banach lattice. Set $X=\left(L_{1}(\mu) \times L_{1}(\mu)\right)^{J}$. We regard $X$ as endowed with the product ordering $\geq_{J}$ given by $\left(a_{j}, b_{j}\right)_{j \in J} \geq_{J}\left(a_{j}^{\prime}, b_{j}^{\prime}\right)_{j \in J}$ if and only if for each $j \in J, a_{j} \geq a_{j}^{\prime}$ and $b_{j} \geq b_{j}^{\prime}$ in $L_{1}(\mu)$. Also, $X$ is regarded as endowed with the product norm $\|\cdot\|_{J}$ given by $\left\|\left(a_{j}, b_{j}\right)_{j \in J}\right\|_{J}=\sum_{j \in J}\left(\left\|a_{j}\right\|_{1}+\left\|b_{j}\right\|_{1}\right)$. Thus $X$ is a Banach lattice. Recall that the Banach lattice $L_{1}(\mu)$ is order continuous and, in particular, Dedekind complete. Hence the same is true for $X$.

Now let $G$ be the subset of $L_{1}(\mu)^{J}$ given by

$$
\begin{array}{r}
G=\left\{\left(h_{j}\right)_{j \in J} \in L_{1}(\mu)^{J}:\right. \\
\text { (ii) and (iii) of the lemma hold relative } \\
\text { to the given family }\left(g_{j}\right)_{j \in J} \in L_{1}(\mu)^{J} \\
\text { and } \left.\sum_{j \in S_{k}} h_{j} \geq-1_{T} \text { for each } k \in K\right\}
\end{array}
$$

and let $H$ be the subset of $X$ given by

$$
H=\left\{\left(h_{j}^{+}, h_{j}^{-}\right)_{j \in J} \in X:\left(h_{j}\right)_{j \in J} \in G\right\} .
$$


Then G is non-empty; e.g. the given family $\left(g_{j}\right)_{j \in J}$ belongs to this set. Hence, $H$ is non-empty also. Let $C$ be a non-empty chain in $H$ for the ordering $\geq_{J}$. Note that $C$ is bounded from below by the zero element of $X$. Hence since $X$ is Dedekind complete, $C$ has an infimum in $X_{+}$, say $\left(a_{j}, b_{j}\right)_{j \in J}$. Evidently, we have $a_{j} \wedge b_{j}=0$ for each $j$. Now since $C$, being a chain, is in particular downwards directed, and since $X$ is order continuous, there is a sequence $\left(\left(h_{j, n}^{+}, h_{j, n}^{-}\right)_{j \in J}\right)_{n=1,2, \ldots}$ in $C$ that converges to $\left(a_{j}, b_{j}\right)_{j \in J}$ in the norm $\|\cdot\|_{J}$ of $X$. In particular, then, $h_{j, n}^{+}-h_{j, n}^{-} \rightarrow a_{j}-b_{j}$ for each $j$ in the norm of $L_{1}(\mu)$. By continuity of addition and of the lattice operations in $L_{1}(\mu)$, it follows that $\left(a_{j}-b_{j}\right)_{j \in J} \in G$ and hence, since $a_{j} \wedge b_{j}=0$ for each $j$, that $\left(a_{j}, b_{j}\right)_{j \in J} \in H$. Thus, every chain for $\geq_{J}$ in $H$ has a lower bound in $H$ for $\geq_{J}$. By Zorn's Lemma, $H$ has a minimal element for $\geq_{J}$. A glance at Lemma 6 shows that if $\left(\underline{h}_{j}^{+}, \underline{h}_{j}^{-}\right)_{j \in J}$ is such a minimal element of $H$, then for some real number $\alpha>0$, (i) of the lemma must hold for the element $\left(\underline{h}_{j}\right)_{j \in J}$ of $G$.

Indeed, let $\left(h_{j}\right)_{j \in J}$ be an element of $G$ for which there is no real number $\alpha>0$ such that $h_{j} \geq-\alpha 1_{T}$ for each $j \in J$. For each $j$, choose a version $\dot{h}_{j}^{+}$of $h_{j}^{+}$ and a version $\dot{h}_{j}^{-}$of $h_{j}^{-}$. Then for almost all $t \in T, \dot{h}_{j}^{+}(t)$ and $\dot{h}_{j}^{-}(t)$ are $\geq 0$ and $\dot{h}_{j}^{+}(t) \wedge \dot{h}_{j}^{-}(t)=0$ for each $j \in J$, and for each $k \in K, \sum_{j \in S_{k}}\left(\dot{h}_{j}^{+}(t)-\dot{h}_{j}^{-}(t)\right) \geq-1$. Modifying the functions $\dot{h}_{j}^{+}$and $\dot{h}_{j}^{-}$on a null set if necessary, we can assume that for each $k \in K, \sum_{j \in S_{k}}\left(\dot{h}_{j}^{+}(t)-\dot{h}_{j}^{-}(t)\right) \geq-1$ holds actually for all $t \in T$. In the same way, we can assume that the other properties hold for all $t \in T$. Then $\sum_{j \in S_{k}}\left(\dot{h}_{j}^{+}-\dot{h}_{j}^{-}\right) \geq-1_{T}$ for each $k \in K, \dot{h}_{j}^{+} \wedge \dot{h}_{j}^{-}=0$ for each $j \in J$, and all the functions $\dot{h}_{j}^{+}$and $\dot{h}_{j}^{-}$are non-negative. Also, it is clear that the condition "there is no real number $\alpha>0$ such that $h_{j} \geq-\alpha 1_{T}$ for each $j \in J$ " means that there is no real number $\alpha>0$ such that for all $j \in J, \dot{h}_{j}^{-}(t) \leq \alpha$ for almost all $t \in T$. Finally, recall that we have $\bigcup_{k \in K} S_{k}=J$ by hypothesis. Consequently, writing $J=\{1, \ldots, n\}$, we may apply Lemma 6 with $\dot{h}_{j}^{+}$in place of $f_{j}$ and $\dot{h}_{j}^{-}$in place of $g_{j}, j=1, \ldots, n$, to find measurable functions $\dot{a}_{j}: T \rightarrow \mathbb{R}_{+}$and $\dot{b}_{j}: T \rightarrow \mathbb{R}_{+}$, $j \in J \equiv\{1, \ldots, n\}$, such that the following properties hold:

(a) $\dot{a}_{j} \leq \dot{h}_{j}^{+}$and $\dot{b}_{j} \leq \dot{h}_{j}^{-}$for all $j \in J$.

(b) For some $j$ there is a $D \in \mathcal{T}$ with $\mu(D)>0$ such that $\dot{a}_{j}(t)<\dot{h}_{j}^{+}(t)$ for all $t \in D$.

(c) $\sum_{j \in S_{k}}\left(\dot{a}_{j}-\dot{b}_{j}\right) \leq \sum_{j \in S_{k}}\left(\dot{h}_{j}^{+}-\dot{h}_{j}^{-}\right)$for each $k \in K$.

(d) $\left(\sum_{j \in S_{k}}\left(\dot{a}_{j}-\dot{b}_{j}\right)\right) \wedge 1_{T}=\left(\sum_{j \in S_{k}}\left(\dot{h}_{j}^{+}-\dot{h}_{j}^{-}\right)\right) \wedge 1_{T}$ for each $k \in K$.

For each $j \in J$ let $a_{j}$ and $b_{j}$ be the elements of $L_{1}(\mu)$ determined by $\dot{a}_{j}$ and $\dot{b}_{j}$, respectively. In particular, we have $a_{j} \geq 0$ and $b_{j} \geq 0$ for each $j \in J$. From (10c) combined with the fact that $\left(h_{j}\right)_{j \in J} \in G$ we see that $\sum_{j \in S_{k}}\left(a_{j}-b_{j}\right) \leq \sum_{j \in S_{k}} g_{j}$ for each $k \in K$, and combining (10d) with the fact that $\left(h_{j}\right)_{j \in J} \in G$ we see 
that $\left(\sum_{j \in S_{k}}\left(a_{j}-b_{j}\right)\right) \wedge 1_{T}=\left(\sum_{j \in S_{k}} g_{j}\right) \wedge 1_{T}$ for each $k \in K$. In particular, for each $k \in K, \sum_{j \in S_{k}}\left(a_{j}-b_{j}\right) \geq-1_{T}$ because $\sum_{j \in S_{k}} g_{j} \geq-1_{T}$ by hypothesis. Thus, the family $\left(a_{j}-b_{j}\right)_{j \in J}$ is an element of $G$. Now from (10a) and the fact that $h_{j}^{+} \wedge h_{j}^{-}=0$ for each $j$, it follows that $a_{j} \wedge b_{j}=0$ for each $j$, and hence that the family $\left(a_{j}, b_{j}\right)_{j \in J}$ is an element of $H$ (since $a_{j}$ and $b_{j}$ are $\geq 0$ for each $j \in J$ ). But (10a) and (10b) together imply that $\left(h_{j}^{+}, h_{j}^{-}\right)_{j \in J} \geq_{J}\left(a_{j}, b_{j}\right)_{j \in J}$ and that $\left(h_{j}^{+}, h_{j}^{-}\right)_{j \in J} \neq\left(a_{j}, b_{j}\right)_{j \in J}$. Thus $\left(h_{j}^{+}, h_{j}^{-}\right)_{j \in J}$ is not a minimal element of $H$ for $\geq_{J}$.

Lemma 8. Let $Z$ be a Riesz space and let $Y$ be an ideal in $Z$. Let $z_{1}, \ldots, z_{n}$ be elements of $Z$ and let $y_{1}, \ldots, y_{n}$ be elements of $Y$. Suppose that $\sum_{i=1}^{n} y_{i} \leq \sum_{i=1}^{n} z_{i}$ and that there is a $y \in Y$ such that $y \leq z_{i}$ for each $i=1, \ldots, n$. Then there are elements $x_{1}, \ldots, x_{n}$ of $Y$ such that $\sum_{i=1}^{n} x_{i}=\sum_{i=1}^{n} y_{i}$ and $x_{i} \leq z_{i}$ for each $i=1, \ldots, n$.

Proof. Let $a=y \wedge y_{1} \wedge y_{2} \wedge \cdots \wedge y_{n}$. For each $i=1, \ldots, n$, set $a_{i}=y_{i}-a$ and $b_{i}=z_{i}-a$. Then for each $i, a_{i} \geq 0$ as well as $b_{i} \geq 0$. Also, $\sum_{i=1}^{n} a_{i} \leq \sum_{i=1}^{n} b_{i}$. The Riesz decomposition theorem provides elements $c_{i} \in Z, i=1, \ldots, n$, such that $\sum_{i=1}^{n} c_{i}=\sum_{i=1}^{n} a_{i}$ and $0 \leq c_{i} \leq b_{i}$ for each $i$. Set $x_{i}=c_{i}+a$ for each $i$. Evidently $\sum_{i=1}^{n} x_{i}=\sum_{i=1}^{n} y_{i}$ and $x_{i} \leq z_{i}$ for each $i$. Also $0 \leq c_{i} \leq \sum_{i=1}^{n} a_{i}$. Now since $y$ and all the $y_{i}$ belong to $Y$ and $Y$ is an ideal in $Z$, we have $a \in Y$ and therefore also $a_{i} \in Y$ for each $i$, and thus $\sum_{i=1}^{n} a_{i} \in Y$, too. Hence, $c_{i} \in Y$ for each $i$ by the facts that $0 \leq c_{i} \leq \sum_{i=1}^{n} a_{i}$ and $Y$ is in ideal in $Z$. We conclude that $x_{i} \in Y$ for each $i$.

\subsection{Proof of Theorem 1}

We first settle some notation. We let $\|\cdot\|_{E}$ denote the norm of $E$ and let $\|\cdot\|_{\Omega}$ denote the product norm on $E^{\Omega}$ given by $\|x\|_{\Omega}=\max \left\{\|x(s)\|_{E}: s \in \Omega\right\}, x \in E^{\Omega}$. Thus the topology on $E^{\Omega}$ given by $\|\cdot\|_{\Omega}$ is the product topology on $E^{\Omega}$ when each factor has the norm topology.

Also we let $w=\sum_{i \in I} w_{i}$ stand for the aggregate endowment. [0,w] denotes the order interval $\left\{x \in E^{\Omega}: 0 \leq x \leq w\right\}$.

Let $\mathcal{H}=\bigcup_{i \in I} \mathcal{H}_{i}$ and let $\mathcal{G}$ be a maximal family of elements of $\mathcal{H}$ such that the indicator functions $1_{S} \in \mathbb{R}^{\Omega}, S \in G$, are linearly independent. In particular, then, since $\mathcal{H}$ and hence $\mathcal{G}$ is finite, we have:

There is a real number $K>0$ such that if $\alpha_{S}, S \in G$, are real numbers

and $\left|\sum_{S \in G} \alpha_{S} 1_{S}(s)\right| \leq 1$ for each $s \in \Omega$ then $\left|\alpha_{S}\right| \leq K$ for all $S \in G$.

Now for each $S \in G$, let $L_{S}=\left\{e 1_{S}: e \in E\right\}$. Then $L_{S}$ is a closed linear subspace of $E^{\Omega}$ for each $S \in G$. We claim that $X_{i} \subset \sum_{S \in G} L_{S}$ for each $i \in I$. To see this, note that by our definition of an economy, each consumption set $X_{i}$ can be written as $X_{i}=\left\{\sum_{S \in \mathcal{H}_{i}} e_{S} 1_{S}: e_{S} \in E_{+}\right\}$. Thus it is enough to show that for any $\bar{S} \in \mathcal{H}$ and any $e \in E, e 1_{\bar{S}} \in \sum_{S \in \mathcal{G}} L_{S}$. Now by choice of $\mathcal{G}$, given $\bar{S} \in \mathcal{H}$ we have 
$1_{\bar{S}}=\sum_{S \in \mathcal{G}} \alpha_{S} 1_{S}$ for some numbers $\alpha_{S}$, whence $e 1_{\bar{S}}=\sum_{S \in \mathcal{G}} \alpha_{S} e 1_{S}$ for any given $e \in E$, and thus $e 1_{\bar{S}} \in \sum_{S \in G} L_{S}$.

For the following let $K$ be a number chosen according to (11). We claim:

$$
\text { If } x=\sum_{S \in \mathcal{G}} x_{S} \text { for } x_{S} \in L_{S} \text { and }\|x\|_{\Omega} \leq 1 \text { then }\left\|x_{S}\right\|_{\Omega} \leq K \text { for each } S \text {. }
$$

Indeed, suppose the condition in (12). Thus $x=\sum_{S \in G} e_{S} 1_{S}$ for points $e_{S} \in E$ by definition of the subspaces $L_{S}$, and $\|x(s)\|_{E} \leq 1$ for each $s \in \Omega$ by choice of $\|\cdot\|_{\Omega}$. Then for any $p \in E^{*}$ with $\|p\|_{E}^{*} \leq 1$ (where $\|\cdot\|_{E}^{*}$ denotes the dual norm of $\|\cdot\|_{E}$ ) and for each $s \in \Omega$

$$
\left|\sum_{S \in \mathcal{G}}\left(p e_{S}\right) 1_{S}(s)\right|=\left|p \sum_{S \in \mathcal{G}} e_{S} 1_{S}(s)\right|=|p x(s)| \leq 1,
$$

and hence from (11), $\left|p e_{S}\right| \leq K$ for each $S \in G$. Consequently $\left\|e_{S}\right\|_{E} \leq K$ for each $S \in G$, whence $\left\|1_{S} e_{S}\right\|_{\Omega} \leq K$ for each $S \in G$, again by choice of $\|\cdot\|_{\Omega}$. Thus (12) holds. (Note that (12) implies that the sum $\sum_{S \in G} L_{S}$ is direct.)

Now consider the order interval $[0, w]$ introduced above and note that $[0, w]$ is the product of the order intervals $[0, w(s)]$ in $E$. Thus, by (A5), $[0, w]$ is $\eta^{\Omega}$-compact. (The topology $\eta^{\Omega}$ was defined prior to the statement of (A5)). Clearly, if $\left(x_{i}\right)_{i \in I}$ is any feasible allocation then $x_{i} \in[0, w]$ for each $i$, and it follows that for each $i \in I$ the set of all the $x_{i}$ that belong to some feasible allocation is $\eta^{\Omega}$-compact. Again by (A5), for each $i \in I$ the preference relation $P_{i}$ has (relatively) $\eta^{\Omega}$-open lower sections. Consequently, for each $i \in I$ the set of all the $x_{i}$ that belong to some feasible and individually rational allocation is $\eta^{\Omega}$-compact, too. By (A3) and the fact that the $P_{i}$ 's have (relatively) $\eta^{\Omega}$-open lower sections, it follows that we can select a finite set $A \subset \bigcup_{i \in I} X_{i}$ such that given any feasible and individually rational allocation $\left(x_{i}\right)_{i \in I}$ there is, for each $i \in I$, a point $x_{i}^{\prime} \in A$ with $x_{i}^{\prime} \in P_{i}\left(x_{i}\right)$.

Let $\mathcal{F}$ be the family of all finite dimensional subspaces $F$ of $E^{\Omega}$ such that

(a) $A \subset F$;

(b) $w_{i} \in F$ for each $i \in I$;

(c) $F=\sum_{S \in G} F_{S}$ for some subspaces $F_{S}$ with $F_{S} \subset L_{S}$ for each $S \in G$.

Then $\mathcal{F}$ is directed by inclusion and since $X_{i} \subset \sum_{S \in \mathcal{G}} L_{S}$ for each $i \in I$, we have that $\mathcal{F}$ is non-empty.

For each $F \in \mathcal{F}$ let $\mathcal{F}^{F}$ be the economy obtained by letting, for each $i \in I$, the consumption set be $X_{i}^{F}=X_{i} \cap F$, the endowment be $w_{i}^{F}=w_{i}$, and the preference relation $P_{i}^{F}$ be the restriction of $P_{i}$ to $X_{i}^{F}$. (That is, if $x \in X_{i}^{F}$ then $P_{i}^{F}(x)=P_{i}(x) \cap F$.) Then, identifying $F$ with some $\mathbb{R}^{\ell}$, each economy $\mathcal{E}^{F}$ satisfies the assumptions of Lemma 2 (observe in particular that the $X_{i}^{F}$ 's are closed in $F$ because, as was noted before, the $X_{i}$ 's are closed in $E^{\Omega}$ ) and thus each $\mathcal{E}^{F}$ has 
an individually rational quasi-equilibrium. That is, for each $F \in \mathcal{F}$ we obtain an allocation $\left(x_{i}^{F}\right)_{i \in I}$ and-using Hahn-Banach extension-an element $p^{F} \in E^{\Omega, *}$ such that

(13c) for each $i \in I$, if $x \in X_{i} \cap F$ satisfies $x \in P_{i}\left(x_{i}^{F}\right)$ then $p^{F} x \geq p^{F} w_{i}$;

$$
w_{i} \notin P_{i}\left(x_{i}^{F}\right) \text { for each } i \in I \text {; }
$$

$$
\left\|p^{F}\right\|_{\Omega}^{*}=1=p^{F} z^{F} \text { for some } z^{F} \in F \text { with }\left\|z^{F}\right\|_{\Omega}=1 .
$$

Since $\mathcal{F}$ is directed by inclusion, the family $\left(\left(x_{i}^{F}\right)_{i \in I}, p^{F}\right)_{F \in \mathcal{F}}$ is a net in $\left(E^{\Omega}\right)^{I} \times E^{\Omega, *}$. Since $\left\|p^{F}\right\|_{\Omega}^{*}=1$ for all $F$, since by (13a), $x_{i}^{F} \in[0, w]$ for each $i$ and all $F$, and since $[0, w]$ is $\eta^{\Omega}$-compact, we can assume, passing to a subnet if necessary, that there is an allocation $\left(x_{i}\right)_{i \in I}$ and a $p \in E^{\Omega, *}$ such that $x_{i}^{F} \rightarrow x_{i}$ in the topology $\eta^{\Omega}$ for each $i \in I$ and $p^{F} \rightarrow p$ weak* in $E^{\Omega, *}$. In particular, then, $\sum_{i \in I} x_{i}=\sum_{i \in I} w_{i}$ by (13a), i.e. the allocation $\left(x_{i}\right)_{i \in I}$ is feasible. (Recall that $\eta$ and hence $\eta^{\Omega}$ are Hausdorff linear topologies.) Moreover, using the fact that by (A5), each $P_{i}$ has (relatively) $\eta^{\Omega}$-open lower sections, we see from (13d) that the allocation $\left(x_{i}\right)_{i \in I}$ is individually rational.

We next show that $p \neq 0$ must hold. Let $|G|$ denote the number of elements of $G$. From (c) of the definition of $\mathcal{F}$ and (13e), for each $F \in \mathcal{F}$ there is an $S \in G$ and a $y^{F} \in L_{S} \cap F$ such that $\left\|y^{F}\right\|_{\Omega} \leq K$ and $p^{F} y^{F} \geq 1 /|G|$ where $K$ is the real number from (12). Passing to a subnet if necessary, we can fix an $\bar{S} \in G$ such that $y^{F} \in L_{\bar{S}}$ for each $F \in \mathcal{F}$. By construction, there exists an $i \in I$ such that every $y \in L_{\bar{S}}$ is $\mathcal{H}_{i}$-measurable. ${ }^{12}$ Thus, by reindexing the consumers if necessary, we may assume that $y^{F}$ is $\mathcal{H}_{1}$-measurable for each $F \in \mathcal{F}$. By assumption (A3), we can select an $\hat{x} \in X_{1}$ such that $\hat{x} \in P_{1}\left(x_{1}\right)$, and since by (A5), $P_{1}$ has (relatively) open upper sections, we may assume $\hat{x} \in \operatorname{int} E_{+}^{\Omega}$. (Indeed, pick any $e \in \operatorname{int} E_{+}$. Then the element $y \in E^{\Omega}$ defined by $y(s)=e$ for each $s \in \Omega$ belongs to int $E_{+}^{\Omega}$ and, being constant across the states, is $\mathcal{H}_{1}$-measurable, thus belonging to $X_{1}$. Consequently, for all $\lambda \in(0,1)$ we have $(1-\lambda) \hat{x}+\lambda y \in X_{1} \cap \operatorname{int} E_{+}^{\Omega}$.)

Now since $\hat{x} \in \operatorname{int} E_{+}^{\Omega}$ and $\left\|y^{F}\right\|_{\Omega} \leq K$ for each $F$, there is a real number $\bar{\gamma}$ such that $\hat{x}-\gamma y^{F} \in \operatorname{int} E_{+}^{\Omega}$ for all $\gamma \in(0, \bar{\gamma})$ and all $F$. Since $y^{F}$ is $\mathcal{H}_{1}$-measurable for each $F$, it follows that $\hat{x}-\gamma y^{F} \in X_{1}$ for all $\gamma \in(0, \bar{\gamma})$ and each $F$. Because $X_{1} \subset \sum_{S \in G} L_{S}$ there is an $F^{1} \in \mathcal{F}$ such that $\hat{x} \in F$ for all $F \in \mathcal{F}$ with $F \supset F^{1}$, and

\footnotetext{
${ }^{12}$ Recall from the beginning of Section 3 that $y \in E^{\Omega}$ being $\mathcal{H}_{i}$-measurable means that $S \in \mathcal{H}_{i}$ and $s, s^{\prime} \in S$ imply $y(s)=y\left(s^{\prime}\right)$.
} 
thus $\hat{x}-\gamma y^{F} \in X_{1} \cap F$ for all $\gamma \in(0, \bar{\gamma})$ and all $F$ with $F \supset F^{1}$. Since $x_{1}^{F} \rightarrow x_{1}$ in the topology $\eta^{\Omega}$ and $\hat{x} \in P_{1}\left(x_{1}\right)$, a glance at assumption (A5) now shows that there are a real number $\beta \in(0, \bar{\gamma})$ and an $F^{2} \in \mathcal{F}$ with $F^{2} \supset F^{1}$ such that $\hat{x}-\beta y^{F} \in P_{1}\left(x_{1}^{F}\right)$ for all $F \in \mathcal{F}$ with $F \supset F^{2}$. Then by (13c), $p^{F}\left(\hat{x}-\beta y^{F}\right) \geq p^{F} w_{1}$. Hence since $p^{F} y^{F} \geq 1 /|G|$ for all $F$, we must have $p^{F} \hat{x} \geq p^{F} w_{1}+\beta /|G|$ for all $F \in \mathcal{F}$ with $F \supset F^{2}$. Because $p^{F} \rightarrow p$ weak* in $E^{\Omega, *}$, it follows that $p \neq 0$, as predicted.

We claim that there is an $i \in I$ such that $p y<p w_{i}$ for some $y \in X_{i}$. Indeed, if for some $i \in I$ and $y \in X_{i}, p y<0$ then it is clear that the claim holds because $X_{i}$ is a cone. Thus suppose that for each $i \in I, p y \geq 0$ for all $y \in X_{i}$. If $p w_{1}>0$-where the index 1 refers to the same agent as above-then again the claim holds. Thus suppose $p w_{1}=0$. Consider the element $\hat{x} \in X_{1}$ from above. Since $p w_{1}=0$, the argument from the previous paragraph implies that $p \hat{x}>0$. Let $\left(x_{i}^{\prime}\right)_{i \in I}$ be a feasible allocation chosen according to (A6). Then, in particular, $x_{1}^{\prime} \in \operatorname{int} E_{+}^{\Omega}$ and hence $\lambda \hat{x} \leq x_{1}^{\prime}$ for $\lambda>0$ but small enough. Thus, for such $\lambda$, $x_{1}^{\prime}-\lambda \hat{x} \in X_{1}$ (since both $x_{1}^{\prime}$ and $\hat{x}$ are $\mathcal{F}_{1}$-measurable) and hence $p\left(x_{1}^{\prime}-\lambda \hat{x}\right) \geq 0$. Consequently $p x_{1}^{\prime}>0$ because $p \hat{x}>0$. Since $\left(x_{i}^{\prime}\right)_{i \in I}$ is feasible, it follows that $p \sum_{i \in I} w_{i}>0$ and hence that $p w_{i}>0$ for some $i \in I$, which establishes the claim.

Pick any $i \in I$ and suppose $y \in X_{i}$ satisfies $y \in P_{i}\left(x_{i}\right)$. Since $X_{i} \subset \sum_{S \in G} L_{S}$ there is an $F^{\prime}$ such that $y \in F$ for all $F \in \mathcal{F}$ with $F \supset F^{\prime}$. Since $x_{i}^{F} \rightarrow x_{i}$ in the topology $\eta^{\Omega}$, assumption (A5) implies that there is an $F^{\prime \prime} \in \mathcal{F}$ such that $y \in P_{i}\left(x_{i}^{F}\right)$ for all $F \in \mathcal{F}$ with $F \supset F^{\prime \prime}$. Consequently, by (13c), $p^{F} y \geq p^{F} w_{i}$ for all $F \in \mathcal{F}$ with $F \supset F^{\prime} \cup F^{\prime \prime}$ whence $p y \geq p w_{i}$ because $p^{F} \rightarrow p$ weak* in $E^{\Omega, *}$. Thus, for each $i \in I$, if $y \in P_{i}\left(x_{i}\right)$ then $p y \geq p w_{i}$.

In particular, then, we must have $p x_{i}=p w_{i}$ for each $i \in I$. Indeed, as noted already, there is an $i \in I$ with $p z<p w_{i}$ for some $z \in X_{i}$. But for such an $i$, " $p y \geq p w_{i}$ for all $y \in P_{i}\left(x_{i}\right)$ " implies that, in fact, $p y>p w_{i}$ for all $y \in P_{i}\left(x_{i}\right)$, since consumption sets are convex and preferences have open upper sections. It follows that the allocation $\left(x_{i}\right)_{i \in I}$ is Pareto optimal. Now since the allocation $\left(x_{i}\right)_{i \in I}$ is both individually rational and Pareto optimal, assumption (A4) and the fact that, for each $i \in I, p y \geq p w_{i}$ whenever $y \in P_{i}\left(x_{i}\right)$ combine to imply that $p x_{i} \geq p w_{i}$ for each $i \in I$, whence $p x_{i}=p w_{i}$ for each $i \in I$ by the feasibility of the allocation $\left(x_{i}\right)_{i \in I}$. We conclude that $\left(\left(x_{i}\right)_{i \in I}, p\right)$ is a non-trivial and individually rational quasi-equilibrium, and thus the theorem is proved.

\subsection{Proof of Theorem $2^{13}$}

Let $\bar{e} \in E_{+}$be chosen according to assumption (A9). We first claim that there is no loss of generality in assuming that $E=L(\bar{e})$. Indeed, let $F$ denote $L(\bar{e})$ endowed with the ordering and the topology induced from $E$; thus $F$ is a locally

\footnotetext{
${ }^{13}$ For the terminology and the facts used in this proof concerning Riesz spaces and Banach lattices, see Aliprantis and Burkinshaw (1985) and Meyer-Nieberg (1991).
} 
convex-solid Riesz space. Note that the corresponding product topology on $F^{\Omega}$ agrees with the topology induced from $E^{\Omega}$. Note also that by choice of $\bar{e}$, we have $L\left(\sum_{i \in I} w_{i}(s)\right)=F$ for each $s \in \Omega$. In particular, for each $i \in I$ the endowment $w_{i}$ belongs to $F^{\Omega}$. Let $\mathcal{E}^{F}$ be the restriction of the economy $\mathcal{E}$ to $F^{\Omega}$.

Suppose $\left(p,\left(x_{i}\right)_{i \in I}\right)$ is a non-trivial quasi-equilibrium for the economy $\mathcal{E}^{F}$. In particular, $p \in F^{\Omega \text {,* }}$. Let $q$ be an extension of $p$ to an element of $E^{\Omega, *}$. Now by (A10), $F$ is dense in $E$ and thus by continuity of the lattice operations in $E$, $F_{+}$is dense in $E_{+}$. By definition of the consumption sets $X_{i}$, this implies that for each $i, X_{i} \cap F_{+}^{\Omega}$ is dense in $X_{i}$. By continuity of preferences in $\mathcal{E}$ (assumption (A5)), it follows that $\left(q,\left(x_{i}\right)_{i \in I}\right)$ is a non-trivial quasi-equilibrium for $\mathcal{E}$.

Next observe that the economy $\mathcal{F}^{F}$ satisfies all the assumption hypothesized for $\mathcal{E}$ in the statement of Theorem 2. Indeed, this is clear for (A1), (A2), (A5), (A9) and (A10). In fact, $\sum_{i \in I} w_{i}(s)$ is an order unit of $F$ for each $s \in \Omega$, so (A10) becomes redundant for $\mathcal{E}^{F}$. Further, since $X_{i} \subset E_{+}^{\Omega}$ for each $i \in I$, and hence every feasible allocation for $\mathcal{E}$ is also a feasible allocation for $\mathcal{F}^{F}$, (A8) must hold for $\mathcal{E}^{F}$. For the same reason, every Pareto optimal and individually rational allocation for $\mathcal{E}^{F}$ also has these properties when regarded as an allocation for $\mathcal{E}$. Consequently, A7) holds for $\mathcal{E}^{F}$ because, as noted above, $X_{i} \cap F_{+}^{\Omega}$ is dense in $X_{i}$ for each $i \in I$. But this latter fact also implies that for each $i \in I$ and $x \in X_{i}$, $P_{i}(x) \cap X_{i} \cap F_{+}^{\Omega}$ is dense in $P_{i}(x)$, because by (A5), $P_{i}(x)$ is (relatively) open in $X_{i}$. Consequently, (A3) and (A4) hold for $\mathcal{E}^{F}$, too.

Thus, as claimed, we can assume in the sequel that $E=L(\bar{e})$. Note that this in particular means that $E=L\left(\sum_{i \in I} w_{i}(s)\right)$ for each $s \in \Omega$ (by the choice of $\bar{e}$ ).

Now since $E$ is a Hausdorff locally convex-solid Riesz space, the positive cone $E_{+}$is closed in $E$ and hence

$$
\|z\|_{\bar{e}}=\inf \left\{\lambda \in \mathbb{R}_{+}:-\lambda \bar{e} \leq z \leq \lambda \bar{e}\right\}
$$

defines an order unit norm on $E \equiv L(\bar{e})$, for which the closed unit ball is

$$
[-\bar{e}, \bar{e}]=\{z \in E:-\bar{e} \leq z \leq \bar{e}\}
$$

In particular, $\bar{e} \in\|\cdot\|_{\bar{e}}$-int $E_{+}$.

In the following, we write $\tau$ for the original topology of $E$. Then $(E, \tau)$ means $E$ regarded as endowed with the topology $\tau$, while $\left(E,\|\cdot\|_{\bar{e}}\right)$ means $E$ regarded as endowed with the topology given by the norm $\|\cdot\|_{\bar{e}}$. Further, $\tau^{\Omega}$ denotes the product topology on $E^{\Omega}$ corresponding to $\tau$.

Observe that Theorem 1 applies to the economy $\mathcal{E}$ with respect to $\left(E,\|\cdot\|_{\bar{e}}\right)$. Indeed, since $(E, \tau)$ is locally convex-solid, $[-\bar{e}, \bar{e}]$ is $\tau$-bounded and therefore the $\|\cdot\|_{\bar{e}^{-}}$-topology is stronger than the topology $\tau$. Hence, assumption (A5) also holds for $\mathcal{E}$ when the original topology $\tau^{\Omega}$ of $E^{\Omega}$ is replaced by the product topology corresponding to $\|\cdot\|_{\bar{e}}$. Let $\left(x_{i}\right)_{i \in I}$ be a feasible allocation chosen according to assumption (A8). Thus for each $i \in I$ and each $s \in \Omega, \sum_{j \in I} w_{j}(s) \leq$ $\lambda x_{i}(s)$ for some real number $\lambda>0$, and hence $\bar{e} \leq \lambda^{\prime} x_{i}(s)$ for some $\lambda^{\prime}>0$ since 
$\bar{e} \in L(\bar{e})=L\left(\sum_{i \in I} w_{i}(s)\right)$ by the choice of $\bar{e}$. Consequently, since $\bar{e}$ belongs to the $\|\cdot\|_{\bar{e}}$-interior of $E_{+}$, so does $x_{i}(s)$ for each $i \in I$ and each $s \in \Omega$. That is, (A6) holds for $\mathcal{E}$ with respect to $\left(E,\|\cdot\|_{\bar{e}}\right)$. Finally, observe that (A4) for the original topology of $E^{\Omega}$ and (A7) combine to imply that (A4) also holds when the original topology $\tau^{\Omega}$ of $E^{\Omega}$ is replaced by the product topology corresponding to $\|\cdot\|_{\bar{e}}$. (To see this, recall the fact that if $C$ is a convex set in a topological vector space $Z$ and $x, y$ are points in $Z$ with $x \in \mathrm{c} \ell C$ and $y \in \operatorname{int} C$, then $(1-\alpha) x+\alpha y \in C$ for $0<\alpha \leq 1$, which implies that $x$ belongs to the closure of $C$ for any linear topology on $Z$.)

Thus (since (A1) to (A3) do not refer to any topology) we may apply Theorem 1 to find a feasible and individually rational allocation $\left(x_{i}\right)_{i \in I}$ together with an $f \in\left(E,\|\cdot\|_{\bar{e}}\right)^{\Omega, *}$ such that $\left(\left(x_{i}\right)_{i \in I}, f\right)$ is a non-trivial quasi-equilibrium, modulo that $f$ need not be continuous for the original topology $\tau^{\Omega}$ of $E^{\Omega}$. We are going to show that there is a $p \in(E, \tau)^{\Omega, *}$ such that the conditions for a non-trivial quasi-equilibrium continue to hold for $\left(\left(x_{i}\right)_{i \in I}, p\right) .{ }^{14}$

In the sequel, if $g$ is a linear functional on $E^{\Omega}$ such that, except $\tau^{\Omega}$-continuity, all conditions of a non-trivial quasi-equilibrium are satisfied for $\left(\left(x_{i}\right)_{i \in I}, g\right)$, we will speak of $\left(\left(x_{i}\right)_{i \in I}, g\right)$ as a non-trivial quasi-equilibrium for simplicity, even though this is not quite in conformity with our definition.

Now because the quasi-equilibrium $\left(\left(x_{i}\right)_{i \in I}, f\right)$ is non-trivial (and since consumption sets are convex and preferences have open upper sections) there is an $i \in I$ for which $y \in P_{i}\left(x_{i}\right)$ actually implies $f y>f w_{i}$. Consequently the allocation $\left(x_{i}\right)_{i \in I}$ is Pareto optimal. Also, by construction, this allocation is individually rational. Thus according to assumption (A4), we have $x_{i} \in \tau^{\Omega}$-c $\ell P_{i}\left(x_{i}\right)$ for each $i \in I$. Moreover, by (A7), for each $i \in I$ there is a convex and $\tau^{\Omega}$-open subset $A_{i}$ of $E^{\Omega}$ such that $\varnothing \neq A_{i} \cap X_{i} \subset P_{i}\left(x_{i}\right)$ and such that $\tau^{\Omega}$-c $\ell P_{i}\left(x_{i}\right) \subset \tau^{\Omega}$-c $\ell A_{i}$ (where we have used the facts that for a convex set $C$ in a topological vector space, int $C$ is convex and, if int $C \neq \varnothing$, then $\mathrm{c} \ell C=\mathrm{c} \ell(\operatorname{int} C))$. Then by the quasi-equilibrium conditions with respect to $\left(\left(x_{i}\right)_{i \in I}, f\right)$, for each $i \in I$ we must have $f x_{i} \leq f y$ for all $y \in A_{i} \cap X_{i}$ (because $f x_{i}=f w_{i}$ by the budget conditions and the feasibility of $\left.\left(x_{i}\right)_{i \in I}\right)$. Note also from above that $x_{i} \in \tau^{\Omega}$-c $\ell A_{i}$ for each $i \in I$.

In view of these facts and since for each $i \in I, X_{i}$ is convex (and $x_{i} \in X_{i}$ ), we may appeal to Lemma 3 to select, for each $i \in I$, a $p_{i} \in(E, \tau)^{\Omega, *}$ and a linear functional $t_{i}$ on $E^{\Omega}$ such that $f=p_{i}+t_{i}, p_{i} x_{i} \leq p_{i} y$ for all $y \in A_{i}$, and $t_{i} x_{i} \leq t_{i} y$ for all $y \in X_{i}$. Since $\tau^{\Omega}$-c $\ell P_{i}\left(x_{i}\right) \subset \tau^{\Omega}$-c $\ell A_{i}$ and $p_{i}$ is $\tau^{\Omega}$-continuous, it follows that for each $i \in I, p_{i} x_{i} \leq p_{i} y$ for all $y \in P_{i}\left(x_{i}\right)$, and since $X_{i}$ is a cone, we must have $t_{i} x_{i}=0$ and $t_{i} y \geq 0$ for all $y \in X_{i}$, whence $p_{i} x_{i}=f x_{i}$ and $p_{i} y \leq f y$ for all $y \in X_{i}$. In particular, $p_{i} w_{i} \leq f w_{i}$ because $w_{i} \in X_{i}$.

\footnotetext{
${ }^{14}$ Note that the price system of a non-trivial quasi-equilibrium must necessarily be $\neq 0$.
} 


\section{We claim:}

If $g$ is any linear functional on $E^{\Omega}$ such that for each $i \in I$, (i) $g y \leq f y$ (14) for all $y \in X_{i}$ but (ii) $g y \geq p_{i} y$ for all $y \in X_{i}$, then $\left(\left(x_{i}\right)_{i \in I}, g\right)$ is again a non-trivial quasi-equilibrium.

Indeed, let $g$ satisfy the conditions in (14), pick any $i \in I$, and let $y \in P_{i}\left(x_{i}\right)$. Then from above, and by the hypothesis that $w_{i} \in X_{i}$,

$$
g y \geq p_{i} y \geq p_{i} x_{i}=f x_{i}=f w_{i} \geq g w_{i}
$$

as well as $g x_{i} \geq p_{i} x_{i} \geq g w_{i}$. Thus for each $i \in I, g x_{i} \geq g w_{i}$ and if $y \in P_{i}\left(x_{i}\right)$ then $g y \geq g w_{i}$. By feasibility of $\left(x_{i}\right)_{i \in I}$ it follows that $g x_{i}=g w_{i}$ for each $i \in I$. Thus $\left(\left(x_{i}\right)_{i \in I}, g\right)$ is a quasi-equilibrium. As for non-triviality, if there is an $i \in I$ such that $g y<0$ for some $y \in X_{i}$ then $\left(\left(x_{i}\right)_{i \in I}, g\right)$ is non-trivial, because $X_{i}$ is a cone. Suppose the other case, i.e. that for each $i \in I, g y \geq 0$ for all $y \in X_{i}$. Then from (14i), for each $i \in I$ and all $y \in X_{i}, f y \geq 0$ as well. But this implies that $f w_{i}>0$ for some $i \in I$ because the quasi-equilibrium $\left(\left(x_{i}\right)_{i \in I}, f\right)$ is nontrivial. From above, $g w_{i}=g x_{i} \geq p_{i} x_{i}=f w_{i}$ and it follows that $\left(\left(x_{i}\right)_{i \in I}, g\right)$ is non-trivial, too.

Now since $\Omega$ is finite, $\left(E,\|\cdot\|_{\bar{e}}\right)^{\Omega, *}$ can be identified with $\left(\left(E,\|\cdot\|_{\bar{e}}\right)^{*}\right)^{\Omega}$ and $(E, \tau)^{\Omega, *}$ can be identified with $\left((E, \tau)^{*}\right)^{\Omega}$ (where $g \in\left(E,\|\cdot\|_{\bar{e}}\right)^{\Omega, *}$ corresponds to $g^{\prime} \in\left(\left(E,\|\cdot\|_{\bar{e}}\right)^{*}\right)^{\Omega}$ if and only if $g x=\sum_{s \in \Omega} g^{\prime}(s) x(s)$ for all $x \in E^{\Omega}$; similarly for $\left.(E, \tau)^{\Omega, *}\right)$. Thus, by the definition of the consumption sets $X_{i}$, the facts that for each $i \in I, f y \geq p_{i} y$ for all $y \in X_{i}$ and $f x_{i}=p_{i} x_{i}$ can be rephrased to state

$$
\sum_{s \in S} p_{i}(s) \leq \sum_{s \in S} f(s) \text { for each } S \in \mathcal{H}_{i} \text { and each } i \in I
$$

and

$$
\sum_{s \in S} p_{i}(s) x_{i}(s)=\sum_{s \in S} f(s) x_{i}(s) \text { for each } S \in \mathcal{H}_{i} \text { and each } i \in I .
$$

(To see this, recall from the definition of the consumption sets $X_{i}$ that $x \in X_{i}$ if and only if $x$ can be written in the form $x=\sum_{S \in \mathcal{H}_{i}} e_{S} 1_{S}$ with $e_{S} \in E_{+}$for each $s \in \Omega$.) Moreover, from (14):

If $h \in\left(\left(E,\|\cdot\|_{\bar{e}}\right)^{*}\right)^{\Omega}$ is such that for each $i \in I, \sum_{s \in S} h(s) \leq \sum_{s \in S} f(s)$ (17) but $\sum_{s \in S} h(s) \geq \sum_{s \in S} p_{i}(s)$ for each $S \in \mathcal{H}_{i}$, then $\left(\left(x_{i}\right)_{i \in I}, h\right)$ is a nontrivial quasi-equilibrium.

Recall that by construction, $\left(E,\|\cdot\|_{\bar{e}}\right)$ is a normed Riesz space such that $\|\cdot\|_{\bar{e}}$ is an order unit norm. An appeal to Lemma 4 and assumption (A5) (order intervals part) reveals that $\left(E,\|\cdot\|_{\bar{e}}\right)$ is in fact a Banach lattice. Thus, in particular, the order dual of $E$ agrees with $\left(E,\|\cdot\|_{\bar{e}}\right)^{*}$. Consequently, $(E, \tau)^{*}$ is an ideal in $\left(E,\|\cdot\|_{\bar{e}}\right)^{*}$ because $(E, \tau)$ is a locally convex-solid Riesz space. (Here and in the 
following, $\left(E,\|\cdot\|_{\bar{e}}\right)^{*}$ is regarded as endowed with the dual ordering relative to the ordering of $E$.)

Fix a $\bar{q} \in(E, \tau)^{*}$, with $\bar{q} \geq 0$ and $\bar{q} \neq 0$, such that $-\bar{q} \leq \sum_{s \in S} p_{i}(s) \leq \bar{q}$ for each $S \in \mathcal{H}_{i}$ and each $i \in I$. (This can be done since $(E, \tau)^{*}$ is a lattice.) Let $B_{\bar{q}}$ be the band in $\left(E,\|\cdot\|_{\bar{e}}\right)^{*}$ generated by $\bar{q}$ and let $B \frac{d}{q}$ be the disjoint complement of $B_{\bar{q}}$ in $\left(E,\|\cdot\|_{\bar{e}}\right)^{*}$, i.e.

$$
B_{\bar{q}}^{d}=\left\{q^{\prime} \in\left(E,\|\cdot\|_{\bar{e}}\right)^{*}: q^{\prime} \perp q \text { for all } q \in B_{\bar{q}}\right\} .
$$

Since $\left(E,\|\cdot\|_{\bar{e}}\right)$ is a Banach lattice, $\left(E,\|\cdot\|_{\bar{e}}\right)^{*}$ is Dedekind complete, and hence $B_{\bar{q}}$ is in fact a projection band, i.e. we have $\left(E,\|\cdot\|_{\bar{e}}\right)^{*}=B_{\bar{q}} \oplus B_{\bar{q}}$. That is, for every $s \in \Omega$ we can write $f(s)=f(s)^{b}+f(s)^{d}$ with $f(s)^{b} \in B_{\bar{q}}$ and $f(s)^{d} \in B \frac{d}{q}$ (the decomposition being unique). For any $i \in I$ and $S \in \mathcal{H}_{i}$, we have $\sum_{s \in S} f(s)^{d} \in B_{\bar{q}}^{d}$ and $\sum_{s \in S} p_{i}(s) \in B_{\bar{q}}$. Hence, from (15), $\sum_{s \in S} f(s)^{d} \geq 0$ and $\sum_{s \in S} f(s)^{b} \geq \sum_{s \in S} p_{i}(s)$. Thus, letting $g$ be the element of $\left(\left(E,\|\cdot\|_{\bar{e}}\right)^{*}\right)^{\Omega}$ defined by $g(s)=f(s)^{b}$ for each $s \in \Omega$, we have

$$
\sum_{s \in S} g(s) \leq \sum_{s \in S} f(s) \text { for each } S \in \mathcal{H}_{i} \text { and each } i \in I
$$

and

$$
\sum_{s \in S} p_{i}(s) \leq \sum_{s \in S} g(s) \text { for each } S \in \mathcal{H}_{i} \text { and each } i \in I \text {. }
$$

In particular, by choice of $\bar{q}$,

$$
\sum_{s \in S} g(s) \geq-\bar{q} \text { for each } S \in \mathcal{H}_{i} \text { and each } i \in I .
$$

Now since $\left(E,\|\cdot\|_{\bar{e}}\right)$ is a Banach lattice with an order unit norm, its dual space $\left(E,\|\cdot\|_{\bar{e}}\right)^{*}$, with the dual norm and ordering, is an $L$-space, and hence so is the band $B_{\bar{q}}$. Thus, having $\bar{q}$ as a weak unit, $B_{\bar{q}}$ is order isomorphic to $L_{1}(\mu)$ on some probability space $(T, \mathcal{T}, \mu)$ such that $\bar{q}$ becomes identified with $1_{T}$. Therefore by Lemma 7 , since $g(s) \in B_{\bar{q}}$ for each $s \in \Omega$, (20) implies that we may find an $h \in\left(\left(E,\|\cdot\|_{\bar{e}}\right)^{*}\right)^{\Omega}$ and a real number $\alpha>0$ such that

$$
\begin{gathered}
h(s) \geq-\alpha \bar{q} \text { for each } s \in \Omega ; \\
\sum_{s \in S} h(s) \leq \sum_{s \in S} g(s) \text { for each } S \in \mathcal{H}_{i} \text { and each } i \in I ; \\
\left(\sum_{s \in S} h(s)\right) \wedge \bar{q}=\left(\sum_{s \in S} g(s)\right) \wedge \bar{q} \text { for each } S \in \mathcal{H}_{i} \text { and each } i \in I .
\end{gathered}
$$

(Note: the set $J$ from Lemma 7 corresponds to $\Omega$, and the family $\left(S_{k}\right)_{k \in K}$ of that lemma to $\bigcup_{i \in I} \mathcal{H}_{i}$.) 
From (21b) and (18) we see that $\sum_{s \in S} h(s) \leq \sum_{s \in S} f(s)$ for each $S \in \mathcal{H}_{i}$ and each $i \in I$, and combining (21c) with (19) and the fact that $\sum_{s \in S} p_{i}(s) \leq \bar{q}$ for each $S \in \mathcal{H}_{i}$ and each $i \in I$, we see that $\sum_{s \in S} h(s) \geq \sum_{s \in S} p_{i}(s)$ for each $S \in \mathcal{H}_{i}$ and each $i \in I$. (Indeed, (19) and $\sum_{s \in S} p_{i}(s) \leq \bar{q}$ together imply that $\sum_{s \in S} p_{i}(s) \leq\left(\sum_{s \in S} g(s)\right) \wedge \bar{q}$, so (21c) yields $\sum_{s \in S} p_{i}(s) \leq\left(\sum_{s \in S} h(s)\right) \wedge \bar{q}$ whence $\sum_{s \in S} p_{i}(s) \leq \sum_{s \in S} h(s)$.) Thus, in view of $(17),\left(\left(x_{i}\right)_{i \in I}, h\right)$ is a nontrivial quasi-equilibrium. If we can show that $h$ actually belongs to $\left((E, \tau)^{*}\right)^{\Omega}$, i.e. is $\tau^{\Omega}$-continuous, then we will have finished the proof.

To this end, recall that $(E, \tau)^{*}$ is an ideal in $\left(E,\|\cdot\|_{\bar{e}}\right)^{*}$, that $\bar{q} \in(E, \tau)^{*}$, and that $p_{i}(s) \in(E, \tau)^{*}$ for each $i \in I$ and each $s \in \Omega$. Consider any $i \in I$ and any $S \in \mathcal{H}_{i}$. Since $\sum_{s \in S} p_{i}(s) \leq \sum_{s \in S} h(s)$, and in view of (21a), we may appeal to Lemma 8 to find elements $q_{i}(s) \in(E, \tau)^{*}, s \in S$, so that $\sum_{s \in S} q_{i}(s)=\sum_{s \in S} p_{i}(s)$ and $q_{i}(s) \leq h(s)$ for each $s \in S$. We must have $q_{i}(s) x_{i}(s)=h(s) x_{i}(s)$ for each $s \in S$. Indeed, by definition of the consumption set $X_{i}$ there is an $e \in E_{+}$such that $x_{i}(s)=e$ for each $s \in S$. Then $q_{i}(s) e \leq h(s) e$ for each $s \in S$. On the other hand, using (16) and the fact that $\sum_{s \in S} h(s) \leq \sum_{s \in S} f(s)$, we have

$\sum_{s \in S} q_{i}(s) e=\left(\sum_{s \in S} q_{i}(s)\right) e=\left(\sum_{s \in S} p_{i}(s)\right) e=\left(\sum_{s \in S} f(s)\right) e \geq\left(\sum_{s \in S} h(s)\right) e=\sum_{s \in S} h(s) e$.

Consequently, $q_{i}(s) e=h(s) e$ for each $s \in S$, i.e. $q_{i}(s) x_{i}(s)=h(s) x_{i}(s)$ for each $s \in S$.

This construction holds for each $S \in \mathcal{H}_{i}$ and $i \in I$. That is, because $\mathcal{H}_{i}$ is a partition of $\Omega$ for each $i \in I$, we have, for each $s \in \Omega$, elements $q_{i}(s) \in(E, \tau)^{*}$ such that for each $i \in I, q_{i}(s) \leq h(s)$ as well as $q_{i}(s) x_{i}(s)=h(s) x_{i}(s)$.

Pick any $s \in \Omega$ and let $q(s)$ be the supremum of $\left\{q_{i}(s): i \in I\right\}$ in $\left(E,\|\cdot\|_{\bar{e}}\right)^{*}$. (This is well defined because $\left(E,\|\cdot\|_{\bar{e}}\right)^{*}$ is the order dual of $E$ as was noted above.) Then $q(s) \leq h(s)$, and since $(E, \tau)^{*}$ is an ideal in $\left(E,\|\cdot\|_{\bar{e}}\right)^{*}$, we have in fact $q(s) \in(E, \tau)^{*}$. Using the Riesz-Kantorovich formula and the facts that $\sum_{i \in I} x_{i}(s)=\sum_{i \in I} w_{i}(s)$ (feasibility) and $\sum_{i \in I} w_{i}(s) \in E_{+}$, we conclude

$$
\begin{aligned}
q(s) \sum_{i \in I} w_{i}(s) & =\sup \left\{\sum_{i \in I} q_{i}(s) y_{i}: y_{i} \in E_{+} \text {and } \sum_{i \in I} y_{i}=\sum_{i \in I} w_{i}(s)\right\} \\
& \geq \sum_{i \in I} q_{i}(s) x_{i}(s) \\
& =\sum_{i \in I} h(s) x_{i}(s) \\
& =h(s) \sum_{i \in I} x_{i}(s) \\
& =h(s) \sum_{i \in I} w_{i}(s) .
\end{aligned}
$$

Consequently $q(s) \sum_{i \in I} w_{i}(s)=h(s) \sum_{i \in I} w_{i}(s)$ because $q(s) \leq h(s)$. Finally, recall from above that $E=L\left(\sum_{i \in I} w_{i}(s)\right)$. Therefore the facts that $q(s) \leq h(s)$ and $q(s) \sum_{i \in I} w_{i}(s)=h(s) \sum_{i \in I} w_{i}(s)$ together imply that $q(s)$ and $h(s)$ agree. It follows that $h \in\left((E, \tau)^{*}\right)^{\Omega}$ and the proof is complete. 


\subsection{Proof of Theorem 3}

With $\bar{e}$ chosen according to Assumption (A9), let $F$ denote the ideal $L(\bar{e})$ with the ordering and the topology induced from $E$. In particular, then, $F^{\Omega}$ is equal to $L\left(\sum_{i \in I} w_{i}\right)$, the order ideal generated by the aggregate endowment $\sum_{i \in I} w_{i}$. Let $\mathcal{E}^{F}$ be the restriction of the economy $\mathcal{E}$ to $F^{\Omega}$. Noting that every feasible allocation for the economy $\mathcal{E}$ is also a feasible allocation for $\mathcal{E}^{F}$, it is readily seen that the economy $\mathcal{E}^{F}$ satisfies all the assumptions of Theorem 2. Thus $\mathcal{F}^{F}$ has a non-trivial quasi-equilibrium $\left(\tilde{f},\left(x_{i}\right)_{i \in I}\right)$; in particular $\tilde{f} \in F^{\Omega, *}$. Using the Hahn-Banach Theorem, let $f$ be an extension of $\tilde{f}$ to an element of $E^{\Omega, *}$. Thus for each $i \in I$,

$$
f x_{i}=f w_{i} \text { and } f y \geq f w_{i} \text { for } y \in P_{i}\left(x_{i}\right) \cap F^{\Omega} .
$$

Note also that according to the proof of Theorem 2, the allocation $\left(x_{i}\right)_{i \in I}$ can be assumed to be Pareto optimal and individually rational for the economy $\mathcal{E}^{F}$, therefore also for the original economy $\mathcal{E}$.

Consider any $i \in I$. By (A4'), $x_{i} \in \mathrm{c} \ell P_{i}\left(x_{i}\right)$, and by (A7'), there is a convex and open subset $A_{i}$ of $E^{\Omega}$ such that $\varnothing \neq A_{i} \cap X_{i} \cap F^{\Omega} \subset P_{i}\left(x_{i}\right) \cap F^{\Omega}$ and such that c $\ell P_{i}\left(x_{i}\right) \subset \mathrm{c} \ell A_{i}$ (using the facts that for a convex set $C$ in a topological vector space, int $C$ is convex and, if int $C \neq \varnothing$, then $\mathrm{c} \ell C=\mathrm{c} \ell$ (int $C$ )). Then $x_{i} \in \mathrm{c} \ell A_{i}$, and from (22), $f x_{i} \leq f y$ for all $y \in A_{i} \cap X_{i} \cap F^{\Omega}$. Since $x_{i} \in X_{i} \cap F^{\Omega}$ and $X_{i} \cap F^{\Omega}$ is convex, we can now use Lemma 3 in a similar way as in the proof of Theorem 2 to find a $p_{i} \in E^{\Omega, *}$ such that $p_{i} x_{i}=f x_{i}, p x_{i} \leq p_{i} y$ for all $y \in P_{i}\left(x_{i}\right)$, and $p_{i} z \leq f z$ for all $z \in X_{i} \cap F^{\Omega}$.

Consider the information partition $\mathcal{H}_{i}$ of agent $i$ and let $\pi_{i}: E^{\Omega} \rightarrow E^{\Omega}$ be the linear operator given by

$$
\pi_{i} y=\sum_{S \in \mathcal{H}_{i}}\left(\frac{1}{\# S} \sum_{s \in S} y(s)\right) 1_{S} \quad \text { for } y \in E^{\Omega},
$$

where \#S stands for the cardinality of the finite set $S$. Then $\pi_{i}$ is a positive projection from $E^{\Omega}$ onto the subspace of $E^{\Omega}$ consisting of the $\mathcal{H}_{i}$-measurable elements of $E^{\Omega}$. Thus, in particular, $\pi_{i} y=y$ for all $y \in X_{i}$. Set

$$
q_{i}=f-f \circ \pi_{i}+p_{i} \circ \pi_{i}
$$

(where $\circ$ means composition of mappings). Then $q_{i} \in E^{\Omega, *}$ since $\pi_{i}$ is linear and continuous. We claim that $f x_{i}=q_{i} x_{i} \leq q_{i} y$ for all $y \in P_{i}\left(x_{i}\right)$ and that $q_{i} z \leq f z$ for all $z \in F_{+}^{\Omega}$. Indeed, since $\pi_{i} y=y$ for $y \in X_{i}$, we have $q_{i} y=p_{i} y$ for $y \in X_{i}$, which, by choice of $p_{i}$, yields the first part of the claim. As for the second, it is evident that if $z \in F_{+}^{\Omega}$ then $\pi_{i} z \in F_{+}^{\Omega}$ by definition of $F$, and thus, in fact, $\pi_{i} z \in F^{\Omega} \cap X_{i}$ because $\pi_{i} z$ is $\mathcal{H}_{i}$-measurable and $X_{i}$ is just the cone of positive $\mathcal{H}_{i}$-measurable elements of $E^{\Omega}$ (by the definition of $X_{i}$ ). But $p_{i} z^{\prime} \leq f z^{\prime}$ for all $z^{\prime} \in F^{\Omega} \cap X_{i}$ by construction, and thus the second part of the claim follows. 
Summing up, for each $i \in I$ there is a $q_{i} \in E^{\Omega, *}$ such that

$$
\begin{gathered}
q_{i} x_{i} \leq q_{i} y \text { for all } y \in P_{i}\left(x_{i}\right) ; \\
q_{i} x_{i}=f x_{i} ; \text { and } \\
q_{i} z \leq f z \text { for all } z \in F_{+}^{\Omega} .
\end{gathered}
$$

Let $q \in E^{\Omega, *}$ be the supremum of the set $\left\{q_{i}: i \in I\right\}$. Observe that $q$ agrees with $f$ on $F^{\Omega}$, i.e. $q z=f z$ for all $z \in F^{\Omega}$. Indeed, by (23c) and the Riesz-Kantorovich formula we have $q z \leq f z$ for $z \in F_{+}^{\Omega}$ because $F^{\Omega}$ is an ideal in $E^{\Omega}$. Using (23b) and the facts that the allocation $\left(x_{i}\right)_{i \in I}$ is feasible, i.e. $\sum_{i \in I} x_{i}=\sum_{i \in I} w_{i}$, and that $x_{i} \geq 0$ for each $i$, another invocation of the Riesz-Kantorovich formula reveals that $q \sum_{i \in I} w_{i} \geq f \sum_{i \in I} w_{i}$. (Cf. the argument at the end of the proof of Theorem 2.) But by construction, $F^{\Omega}$ is equal to $L\left(\sum_{i \in I} w_{i}\right)$, and it follows that $q$ and $f$ agree on $F^{\Omega}$, as predicted.

Now by the facts that $q \geq q_{i}$ and $P_{i}\left(x_{i}\right) \subset E_{+}^{\Omega}$ for each $i \in I$, (23a) and (23b) imply that for each $i \in I, q y \geq f x_{i}$ whenever $y \in P_{i}\left(x_{i}\right)$. But from this and the facts that $\left(\tilde{f},\left(x_{i}\right)_{i \in I}\right)$ is a non-trivial quasi-equilibrium for $\mathcal{E}^{F}$ and that, on $F^{\Omega}, q$ agrees with $f$ and hence with $\tilde{f}$ (by construction of $f$ ) it is plain that $\left(q,\left(x_{i}\right)_{i \in I}\right)$ is a non-trivial quasi-equilibrium for $\mathcal{E}$. This completes the proof of the theorem.

\section{References}

AlipRANTIS, C. D. AND O. BURKINSHAW (1985): Positive Operators, no. 119 in Pure and Applied Mathematics Series, London, Orlando: Academic Press.

Aliprantis, C. D., R. Tourky, AND N. C. YANnelis (2000): "Cone Conditions in General Equilibrium Theory,” Journal of Economic Theory, 92, 96-121.

- - (2001): "A Theory of Value with Non-Linear Prices: Equilibrium Analysis beyond Vector Lattices,” Journal of Economic Theory, 100, 22-72.

AlLEN, B. (2005): "Market Games with Asymmetric Information: The Core," Economic Theory, (forthcoming).

ARAUJO, A. P. AND P. K. MONTEIRO (1989): "Equilibrium without Uniform Conditions," Journal of Economic Theory, 48, 416-427.

BEWLEY, T. F. (1972): "Existence of Equilibria in Economies with Infinitely Many Commodities," Journal of Economic Theory, 4, 514-540.

FlORENZANO, M. (1983): "On the Existence of Equilibria in Economies with an Infinite Dimensional Commodity Space,” Journal of Mathematical Economics, 207-220. 
GALE, D. AND A. MAs-Colell (1975): "An Equilibrium Existence Theorem for a General Model without Ordered Preferences," Journal of Mathematical Economics, 2, 9-16.

Glycopantis, D., A. Muir, AND N. C. YANNELIS (2003): "On Extensive Form Implementation of Contracts in Differential Information Economies," Economic Theory, 21, 495-526.

Glycopantis, D. AND N. C. YANNElis, eds. (2005a): Differential Information Economies, Berlin Heidelberg New York: Springer Verlag.

GLYCOPANTIS, D. AND N. C. YANNELIS (2005b): "Equilibrium Concepts in Differential Information Economies," in Differential Information Economies, ed. by D. Glycopantis and N. C. Yannelis, Berlin Heidelberg New York: Springer Verlag.

Hervés-Beloso, C., E. Moreno-GARCíA, AND N. C. YANNelis (2005): “Characterization and Incentive Compatibility of Walrasian Expectations Equilibrium in Infinite Dimensional Commodity Spaces," Economic Theory, 26, 361-381.

KrASA, S. AND N. C. YANNELIS (1994): "The Value Allocation of an Economy with Differential Information,” Econometrica, 62, 881-900.

Lefebvre, I. (2001): “An Alternative Proof of the Non-Emptiness of the Private Core," Economic Theory, 18, 275-291.

MAS-Colell, A. (1986): "The Price Equilibrium Existence Problem in Topological Vector Lattices,” Econometrica, 54, 1039-1054.

MeYer-NieberG, P. (1991): Banach Lattices, New York: Springer-Verlag.

PAGE, F. (1997): "Market Games with Differential Information and Infinite Dimensional Commodity Spaces: The Core,” Economic Theory, 9, 151-159.

PoDCZECK, K. (1996): "Equilibria in Vector Lattices without Ordered Preferences or Uniform Properness," Journal of Mathematical Economics, 25, 465-485.

RADNER, R. (1968): “Competitive Equilibrium under Uncertainty,” Econometrica, $36,31-58$.

SHAFER, W. J. (1976): "Equilibrium in Economies without Ordered Preferences or Free Disposal," Journal of Mathematical Economics, 3, 135-137.

Tourky, R. (1998): "A New Approach to the Limit Theorem on the Core of an Economy in Vector Lattices," Journal of Economic Theory, 78, 321-328.

-_- (1999): "The Limit Theorem on the Core of a Production Economy in Vector Lattices with Unordered Preferences,” Economic Theory, 14, 219-226. 
Won, D. C. AND N. C. YANNELIS (2005): "Equilibrium Theory with Unbounded Consumption Sets and Non-Ordered Preferences, Part I: Non-Satiation,” Tech. rep., University of Illinois at Urbana Champaign.

YANNELIS, N. C. (1991): "The Core of an Economy with Differential Information,” Economic Theory, 1, 183-198.

YANNELIS, N. C. AND W. R. ZAME (1986): "Equilibria in Banach Lattices without Ordered Preferences,” Journal of Mathematical Economics, 85-110. 